\title{
A Quantitative Map of the Circuit of Cat Primary Visual Cortex
}

\author{
Tom Binzegger, ${ }^{1,2}$ Rodney J. Douglas, ${ }^{1}$ and Kevan A. C. Martin ${ }^{1}$ \\ ${ }^{1}$ Institute of Neuroinformatics, University of Zürich, and Eidgenössische Technische Hochschule Zürich, CH-8057 Zürich, Switzerland, and ${ }^{2} \mathrm{Hen} r y$ \\ Wellcome Building for Neuroecology, University of Newcastle upon Tyne, Newcastle upon Tyne NE2 4HH, United Kingdom
}

\begin{abstract}
We developed a quantitative description of the circuits formed in cat area 17 by estimating the "weight" of the projections between different neuronal types. To achieve this, we made three-dimensional reconstructions of 39 single neurons and thalamic afferents labeled with horseradish peroxidase during intracellular recordings in vivo. These neurons served as representatives of the different types and provided the morphometrical data about the laminar distribution of the dendritic trees and synaptic boutons and the number of synapses formed by a given type of neuron. Extensive searches of the literature provided the estimates of numbers of the different neuronal types and their distribution across the cortical layers. Applying the simplification that synapses between different cell types are made in proportion to the boutons and dendrites that those cell types contribute to the neuropil in a given layer, we were able to estimate the probable source and number of synapses made between neurons in the six layers. The predicted synaptic maps were quantitatively close to the estimates derived from the experimental electron microscopic studies for the case of the main sources of excitatory and inhibitory input to the spiny stellate cells, which form a major target of layer 4 afferents. The map of the whole cortical circuit shows that there are very few "strong" but many "weak" excitatory projections, each of which may involve only a few percentage of the total complement of excitatory synapses of a single neuron.
\end{abstract}

Key words: cell types; cell reconstruction; interlaminar connectivity; synaptic weights; connectivity rule; laminar distribution

\section{Introduction}

One of the most difficult problems faced by neuroscientists is how to trace the circuits of complex networks like the neocortex. There have long been effective techniques for revealing the projection pathways (i.e., the entire dendritic and axonal trees) of individual neurons and now increasingly sophisticated labeling methods can be applied to reveal groups of neurons in the circuit with common characteristics, like the same neurotransmitter, or that they project to the same nucleus. The difficulty remains in establishing who talks to whom and how much in a cortical circuit built up of many thousands of neurons. The time-honored technique of circuit building is to assume that axons connect only to the neurons of which the somata lie in the layer of axonal arborization (Lorente de Nó, 1949; Lund et al., 1979; Gilbert, 1983). Using this method, one of the most complete cortical circuits for excitatory neurons was proposed for cat visual cortex by Gilbert and Wiesel (1981) from data obtained by intracellularly labeling neurons in cat visual cortex in vivo. Their circuit was consistent with that conjectured from previous studies of cortical receptive fields (Hubel and Wiesel, 1962, 1965). Subsequent experimental work by Gilbert and collaborators provided additional support for aspects of their circuit (McGuire et al., 1984; Bolz and Gilbert, 1986; Bolz et al., 1989).

The problem of generating a qualitative schematic of the cor-

Received April 14, 2004; revised July 28, 2004; accepted July 28, 2004.

This work was supported by European Union Grant QULG3-1999-01064 and Human Frontier Science Program Grant RG0123/2000-B to K.A.C.M. T.B. was also supported in part by the Wellcome Trust. We thank John Anderson for being ever-present.

Correspondence should be addressed to Tom Binzegger, School of Biology, Henry Wellcome Building for Neuroecology, University of Newcastle upon Tyne, Newcastle upon Tyne NE2 4HH, UK. E-mail: tom.binzegger@ncl.ac.uk. DOI:10.1523/JNEUROSCI.1400-04.2004

Copyright $\odot 2004$ Society for Neuroscience $\quad$ 0270-6474/04/248441-14\$15.00/0 tical circuit is greatly simplified if an individual neuron receives all of its connections essentially from a single source, as Gilbert and Wiesel for the most part assumed. But a feature of cortical neurons is that they are polyneuronally innervated; their excitatory and inhibitory synapses arise from multiple sources (Ahmed et al., 1994). If polyneuronal innervation is to be incorporated properly, the relative anatomical "weight" must also be assigned to a given connection. These weights are rarely considered, but when they are, the results have been surprising; for example, Ahmed et al. $(1994,1997)$ physically mapped the synapses on the dendritic trees of identified spiny stellate and basket cells in layer 4 of the cat primary visual cortex and found that only $\sim 5 \%$ of the excitatory synapses arose from the lateral geniculate nucleus (LGN). Their experimental estimates of the thalamic component were in good agreement with theoretical estimates of Peters and Payne (1993).

The approximation used by Peters and Feldman (1976) and Peters and Payne (1993) is now referred to as Peters's rule (Braitenberg and Schüz, 1991). They assumed that axons simply connect in direct proportion to the occurrence of all synaptic targets in the neuropil. The rule can be extended to provide more precise estimates by taking into account the detailed morphometrics of the dendritic and axonal trees of the different neuronal types. For example, in the case of a deep layer pyramidal cell, of which the apical dendrite may span several layers, one could include in the calculation the additional synapses formed on the apical dendrite in each layer. However, this cannot be done, because comprehensive data on the comparative morphometrics of the dendritic and axonal trees for different types of neurons is lacking. In the present study, we surmounted this major obstacle by making detailed three-dimensional reconstructions and measurements of representative cortical neurons of cat area 17 to provide much of the missing quantitative data. Combined with 
published data, we produced a comprehensive quantitative estimate of the map of the local neuronal circuit of cat visual cortex.

\section{Materials and Methods}

Preparation and maintenance of animals

The neurons examined in this study were obtained from anesthetized adult cats that had been prepared for in vivo intracellular recording (Martin and Whitteridge, 1984) (for details, see Douglas et al., 1991). All experiments were performed under the authorization of animal research licenses granted to K.A.C.M. by the Home Office of the United Kingdom and the Cantonal Veterinary Authority of Zürich. We first recorded from each cell extracellularly and mapped the receptive field orientation preference, size, type, binocularity, and direction preference by hand. The mapping was repeated intracellularly, and horseradish peroxidase (HRP) was then ionophoresed into the cell. Thalamic afferents were classified as X- or Y-like using a battery of tests (Friedlander and Stanford, 1984; Martin and Whitteridge, 1984). After appropriate survival times, the brains were fixed. The block of tissue containing the intracellularly filled neurons was serially sectioned in the coronal plane at a thickness of 80 $\mu \mathrm{m}$ and processed to reveal the HRP and osmicated and embedded in resin to reduce differential shrinkage. Shrinkage of the tissue was estimated to be $11 \%$. This processing allowed the material to be examined at both the light and electron microscopic level.

\section{Cell reconstructions}

Neurons were reconstructed in three dimensions with the aid of a light microscope (Leitz Dialux 22; Leitz, Wetzlar, Germany) and drawing tube attached to an in-house three-dimensional reconstruction system (Botha et al., 1987). Neurons were reconstructed at $400 \times$ magnification. The reconstructions were characterized by a list of data points and stored for additional usage. Each data point consists of a code describing the digitized structure (axon, bouton, or dendrite) and its three spatial coordinates and thickness (where relevant). Axonal and dendritic collaterals are represented by open, piecewise, linear curves. The axonal arborizations are complex and often extend through many histological sections. The measurement error of the digitized structures was estimated by measuring four boutons 10 times. The SD was smaller than $0.6 \mu \mathrm{m}$ in all three dimensions.

\section{Laminar distribution of boutons and dendritic trees}

For each section, the affiliation of the boutons, axonal and dendritic segments in the section to one of the five cortical layers 1, 2/3 (i.e., layers 2 and 3 where combined), 4, 5, and 6, was determined using the lamina border criteria of Henry et al. (1979). The relative position of the laminar borders and the boutons and axonal and dendritic trees of all sections was summarized in a single coronal projection (see Fig. 3). In general, the lamina borders of this summary diagram are distorted and does not show the correct layer thickness. In particular, for those cells in which the axon extended over large ( $>1 \mathrm{~mm}$ ) anteroposterior distances, it was not possible to represent in the single coronal projection both the correct relationship between the reconstructed neuron and laminas boundaries and the correct location of the boundaries.

Based on the summary diagram (see Fig. 3), it was straightforward to count the number of boutons that a reconstructed neuron formed in each layer. For an estimate of the summed length of the part of the dendritic trees that a neuron formed in each layer, we divided the piecewise linear curves representing the dendritic collaterals in the three dimensional space into segments of $1 \mu \mathrm{m}$ length and counted the number of segments in each cortical layer.

\section{Basic properties of cortical layers}

Total number of neurons and synapses per cortical layer. Beaulieu and Colonnier (1983) counted for each cortical layer the number of cell bodies under a square millimeter of cortical surface in cat area 17. Multiplying these numbers with the total surface area of one cortical hemisphere, which for the cat is $399 \mathrm{~mm}^{2}$ (Anderson et al., 1988), we obtained the total number of neurons per layer. The proportion of GABAergic [i.e., smooth $(\mathrm{sm})]$ neurons for each layer was estimated by Gabbott and Somogyi (1986). Thus, the total number of neurons is $0.50 \times 10^{6}$ (of which $97 \%$ are GABAergic) for layer $1,10.58 \times 10^{6}(22 \%$ GABAergic $)$ for layer $2 / 3,10.93 \times 10^{6}(20 \%$ GABAergic $)$ for layer 4, $2.36 \times 10^{6}(18 \%$ GABAergic) for layer 5, and $6.92 \times 10^{6}(17 \%$ GABAergic $)$ for layer 6 .

The number of asymmetric and symmetric synapses under a square millimeter of cortical surface was counted for each cortical layer in cat area 17 by Beaulieu and Colonnier (1985). Again, we multiplied these numbers by the total cortical surface area. The resulting total number of synapses is $21.54 \times 10^{9}$ (of which $11.5 \%$ are symmetric) for layer 1 , $57.93 \times 10^{9}(15.8 \%$ symmetric $)$ for layer $2 / 3,61.32 \times 10^{9}(15.8 \%$ symmetric) for layer $4,15.36 \times 10^{9}(15.8 \%$ symmetric $)$ for layer 5 , and $22.93 \times 10^{9}(16.2 \%$ symmetric $)$ for layer 6 .

\section{Peters's rule}

Let $S_{j}^{u}$ be the number of synapses in cortical layer $u$ (of cat area 17) formed by the presynaptic neurons of type $j$ and $N^{u}$ the number of neurons in layer $u$. In its simplest form, Peters's rule states that the $S_{j}^{u}$ synapses distribute evenly over the $N^{u}$ potential target neurons (i.e., each neuron in layer $u$ receives $S_{j}^{u} / N^{u}$ synapses of type $j$ ). However, this rule ignores some known facts about cortical connectivity. First, not every cell type in layer $u$ necessarily receives synapses of type $j$. An example is the chandelier cells that form synapses with pyramidal cells only. Second, the synapses of type $j$ can also contact neurons of which the cell bodies are in another layer but form dendrites in layer $u$. Third, the number of synapses per neuron in layer $u$ could vary depending on the type of the postsynaptic neuron. Such variations could be caused by size differences of the dendritic trees, by particular preferences of these synapses on the dendritic trees (i.e., proximal region or distal region), or by variations of the synaptic density along dendrites. We therefore generalized Peters's rule to account for some of these properties.

We assume that each neuron can form synapses with the dendritic trees, the axonal tree (in the case of the chandelier cells), or the cell body of a postsynaptic target neuron. For each pair of cell types $j$ (presynaptic) and $i$ (postsynaptic), we therefore introduce the following two quantities: $p_{i j}^{u}$ and $\rho_{j}^{u} . p_{i j}^{u}$ is the typical length of the dendritic (or axonal) processes located in layer $u$ of the neurons of type $i$ that are able to receive synapses of type $j$ ("potential target processes"). $\rho_{j}^{u}$ is the proportion of the synapses $S_{j}^{u}$ that contact cell bodies in layer $u\left(\rho_{j}^{\mathrm{u}} \in[0,1]\right)$. We assume again that the total number of synapses of type $j$ devoted to the potential target processes [i.e., $\left(1-\rho_{j}^{u}\right) S_{j}^{u}$ ] distribute evenly among the potential target processes in layer $u$, and that the synapses devoted to cell bodies (i.e., $\rho_{j}^{u} S_{j}^{u}$ ) distribute evenly among the cell bodies in layer $u$. Thus, each individual neuron of type $i$ receives in layer $u$ an (average) number of synapses:

$$
\bar{s}_{i j}^{u}=\left(1-\rho_{j}^{u}\right) S_{j}^{u} \cdot \frac{p_{i j}^{u}}{\sum_{k} n_{k} p_{k j}^{u}}+\rho_{j}^{u} S_{j}^{u} \cdot \frac{\delta_{i}^{u}}{N^{u}},
$$

from all of the neurons of type $j$, where $n_{k}$ is the number of neurons of type $k$. The sum $\Sigma_{k} n_{k} p_{k j}^{u}$ is the total length of all of the potential processes in layer $u$ available to form synapses with neurons of type $j . \delta_{i}^{u}$ is 1 if cell type $i$ is located in layer $u$; otherwise, it is zero. This term indicates that synapses in layer $u$ cannot contact cell bodies outside layer $u$. Equation 1 reduces to the simple form of Peters's rule if the typical length of the processes in layer $u$ that are able to receive synapses from neurons of type $j$ is similar for all neurons in the layer, and no cell type located outside layer $u$ forms such processes in layer $u$ (i.e., $p_{k j}^{u}=\delta_{k}^{u} p^{u}$ for some $p^{u}>0$ ).

Synapses made by chandelier cells $\left(\mathrm{j}=\mathrm{j}_{\text {axo } 2 / 3}\right)$

Chandelier cells make symmetric synapses with the axonal initial segment of pyramidal cells (Somogyi et al., 1982). We assume that the chandelier cells and their axons are located primarily in layers 2/3 (Fairen and Valverde, 1980; Somogyi et al., 1982), and we ignore synapses formed in the remaining layers. The synapses formed by the chandelier cells $\left(S_{j_{\mathrm{axo} 2 / 3}^{u}}\right)$ distribute on the axon initial segments of the pyramidal cells in layer $2 / 3\left(i_{p 2 / 3}\right)$. We denote the average length of these segments by $a$. We therefore have $p_{i j_{\mathrm{xx} 2 / 3}^{u}}^{u}=a$ if $u$ is layer $2 / 3$ and $i=i_{p 2 / 3}$, and $p_{i j_{\mathrm{ax} 2 / 3}}^{u}=0$ otherwise. Using Equation 1, it follows with $\rho_{j_{\text {axo } 2 / 3}^{u}}^{u}=0$ :

$$
\bar{s}_{i j_{a x a 2 / 3}}^{u}= \begin{cases}S_{j_{a x o 2 / 3}^{u}}^{u} / n_{i} & \text { if } u \text { is layer } 2 / 3 \text { and } i=i_{p 2 / 3} \\ 0 & \text { otherwise. }\end{cases}
$$


Anatomical studies indicate that the total number of synapses formed by the chandelier cells on the axon initial segment is between 20 and 43 (Somogyi et al., 1982; Farinas and DeFelipe, 1991). Here, we use the mean value (i.e., $\bar{s}_{i_{p 2 / 3 \mathrm{j} \times 02 / 3}^{u}}=32$ ). Note that to be consistent with Equation 2, the frequency of chandelier cells in layer $2 / 3$ must be $n_{j_{a x 02 / 3}}=$ $8 \times 10^{4}$ (i.e., $\sim 3.4 \%$ of the number of smooth neurons in layer $2 / 3$ ). Indeed, $n_{i_{2 / 3}}=8.2 \times 10^{6}$ (Beaulieu and Colonnier, 1983; Gabbott and Somogyi, 1986), and $S_{j_{\text {axo } 2 / 3}^{u}}=3300 \times n_{j_{a x 02 / 3}}$ because a chandelier cell forms $\sim 3300$ synapses in layer 2/3 (Somogyi et al., 1982; Freund et al., 1983).

Synapses made by remaining cell types $\left(\mathrm{j} \neq \mathrm{j}_{\text {axo } 2 / 3}\right)$

To compute $\bar{s}_{i j}^{u}$ for the remaining pairs of cell types (i.e., $j \neq j_{a x o 2 / 3}$ ), we assume that the synapses $\left(1-\rho_{j}^{u}\right) S_{j}^{u}$ are distributed on all dendrites in this layer without any restrictions. Thus, we have $p_{i j}^{u}=d_{i}^{u}$ where $d_{i}^{u}$ is the average length of the dendrites in layer $u$ formed by the neurons of type $i$. Therefore, Equation 1 takes the following form:

$$
\bar{s}_{i j}^{u}=\left(1-\rho_{j}^{u}\right) S_{j}^{u} \frac{d_{i}^{u}}{\sum_{k} n_{k} d_{k}^{u}}+\rho_{j}^{u} S_{j}^{u} \frac{\delta_{i}^{u}}{N^{u}} .
$$

$\Sigma n_{k} d_{k}^{u}$ is the summed length of all dendrites in layer $u$.

The three-dimensional reconstructions were used to estimate the average dendritic length $d_{k}^{u}$ and the average number of synapses (denoted by $\bar{s}_{k}^{u}$ ) that the neuron of each cell type $k$ form in layer $u$. The third parameter we have to estimate is $n_{k}$, the number of neurons per cell type. These estimates were obtained from the literature. The total number of synapses formed in layer $u$ by a cell type follows from $S_{j}^{u}=n_{j} \bar{s}_{j}^{u}$.

With the exception of the basket cells, we set $\rho_{j}^{u}=0$ (i.e., we assume that basket cells are the only neurons in area 17 that form synapses with cell bodies). The proportion of boutons of which the postsynaptic targets are cell bodies is indeed very small for spiny cells (McGuire et al., 1984; Kisvárday et al., 1986; Gabbott et al., 1987; Somogyi, 1989; Anderson et al., 1994a), double bouquet cells (Somogyi and Cowey, 1981; Freund et al., 1986; Tamás et al., 1997, 1998), dendrite targeting cells (Somogyi and Cowey, 1981; Freund et al., 1986; Tamás et al., 1997, 1998), and thalamic afferents (Garey and Powell, 1971; McGuire et al., 1984; Freund et al., 1985). We set $\rho_{j}^{u}=0.47$ for the basket cells in layer $2 / 3$ (Freund et al., 1986; Tamás et al., 1997, 1998), $\rho_{j}^{u}=0.30$ for the basket cell in layer 4 (Somogyi et al., 1983; Kisvárday et al., 1985), and $\rho_{j}^{u}=0.20$ for the basket cells in layer 5 (Kisvárday et al., 1987).

\section{Perturbation of the proportion of neurons}

We denote by $\alpha_{j_{0}}$ the proportion of excitatory (inhibitory) neurons in layer $u$ that belong to an arbitrary but fixed excitatory (inhibitory, respectively) cell type $j_{0}$. We perturb the proportion $\alpha_{\mathrm{j}_{0}}$ without affecting the total number of excitatory and inhibitory neurons in this layer. Thus, if $\alpha_{j_{0}}(\Delta \alpha)=\alpha_{j_{0}}+\Delta \alpha$ is the perturbed proportion of neurons of type $j_{0}$ ( $\Delta \alpha$, such that $\left.0<\alpha_{j_{0}}+\Delta \alpha<1\right)$, we also have to change the proportion $\alpha_{\mathrm{j}}$ of neurons belonging to the remaining excitatory (inhibitory) cell types $j$ of layer $u$. This is achieved, for example, by setting the following:

$$
\alpha_{j}(\Delta \alpha)=\left(1-\alpha_{j_{0}}-\Delta \alpha\right) \frac{n_{j}}{M^{u}-n_{j}},
$$

where $M^{u}$ is the total number of excitatory (inhibitory) neurons in layer $u$. In particular, for this choice, the ratio of the perturbed cell numbers remains unchanged (i.e., with $n_{j}(\Delta \alpha)=\alpha_{j}(\Delta \alpha) M^{u}$, we have $\frac{\mathrm{n}_{\mathrm{j}}(\Delta \alpha)}{\mathrm{n}_{\mathrm{k}}(\Delta \alpha)}=\frac{\mathrm{n}_{\mathrm{j}}}{\mathrm{n}_{\mathrm{k}}}$ for all excitatory (inhibitory) cell types $j$ and $k$ in layer $u$ that are different from $j_{0}$ ).

\section{Results}

We estimated the average number of synapses formed between neurons in different layers using the extension of Peters's rule (see Materials and Methods) (Fig. 1). To do so, we needed to estimate three sets of parameters (i.e., the laminar patterns of the synaptic "clouds" and the dendritic trees) and the number of neurons of each cell type.

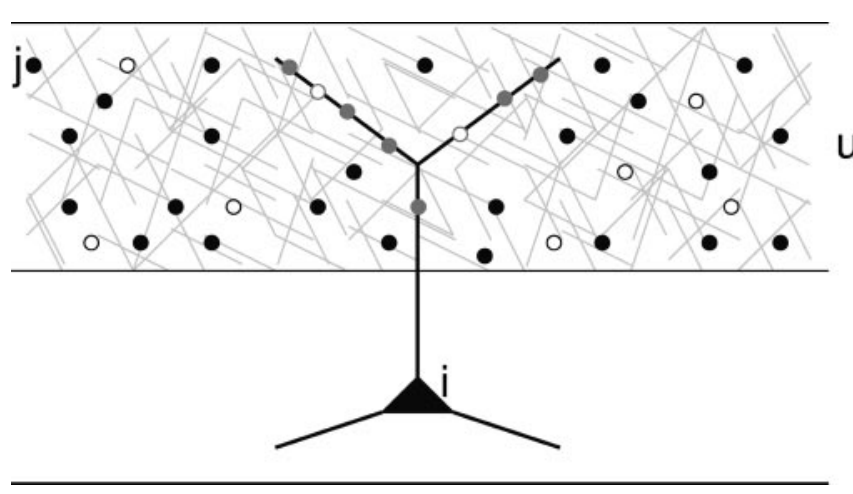

Figure 1. Peters's rule. Depicted is the situation where a typical neuron of cell type $i$ has its apical dendrite in layer $u$ (indicated by black lines). Neurons innervating this layer form synapses (indicated by closed and open circles) with the dendritic branches in this layer (indicated by gray lines), including the apical dendrite of neuron $i$ (indicated by closed and open gray dots). Synapses belonging to the neurons of cell type $j$ are indicated by closed dots. In this instance, Peters's rule specifies the number of synapses that all neurons of cell type $j$ form with the apical dendrite of neuron $i$ (the closed gray dots) according to the following equation: $s_{i j}^{u}=S_{j}^{u} d_{i}^{u} / D^{u}$. Here, $S_{j}^{u}$ is the total number of synapses in layer $u$ that are formed by the neurons of typej (i.e., the number of closed dots). $D^{u}$ is the summed length of all dendritic branches in layer $u$ (i.e., the sum of the length of all gray lines and the apical dendrite indicated in black), and $d_{i}^{u}$ is the (average) length of the dendrites formed by the neurons of type $i$ in layer $u$ (i.e., the sum of the branches of the black apical dendrite). Intuitively, the $S_{j}^{u}$ synapses are evenly distributed about all dendritic segments in the layer, so that the number of synapses per dendritic length is $S_{j}^{u} / D^{u}$. Multiplying with the length $d_{i}^{u}$ results in the number of synapses that are formed with the emphasized black apical dendrite.

\section{Laminar patterns of synapses}

We analyzed the laminar pattern of synaptic boutons of 39 reconstructed neurons. The cells were classified into conventionally recognized cell types, which are defined according to anatomical features such as the laminar location of the cell body, whether the dendrites are smooth or spiny, and the branching characteristics of the axonal and dendritic trees (O'Leary, 1941; Lorente de Nó, 1949; Szentagothai, 1973; Szentagothai and Arbib, 1974; Lund et al., 1979; Ramon y Cajal, 1988). The range of cell types present in the database of reconstructed neurons is shown in Table 1 and Figure 2. Spiny neurons are well represented in the database and, with the exception of the spiny neurons in layer 1, we have at least one reconstructed example for each major spiny cell type. Thalamic afferents, which form the major extra-striate source of asymmetric synapses in layer 4 (Ahmed et al., 1994), are also included. Anatomical studies of cortical smooth neurons indicate that, like spiny neurons, a variety of types exist (Peters and Regidor, 1981). For one of the most prominent, the basket cells, we have samples from layer $2 / 3,4$, and 5 . In addition, we reconstructed a double bouquet cell from layer $2 / 3$. For other types, of which we had no reconstructions (e.g., chandelier cells, Martinotti cells, or Cajal-Retzius cells), we assumed average dimensions for their dendritic trees and obtained bouton distributions and numbers from published work.

The laminar pattern of synapses cannot be determined directly from the reconstructions, because synapses can only be detected by electron microscopy. However, from extensive combined light and electron microscopy, we could be sure that synapses are associated almost invariably with axonal boutons, which are visible with the light microscope and thus could be counted for all neurons (Fig. 3, white dots). The number of synapses per bouton varies between cell types but is generally close to one (Fig. 4 for exact numbers and references). To determine the number of synapses per layer, we first counted the number of boutons per layer and then multiplied these numbers by the 


\section{Table 1. List of reconstructed neurons}

\begin{tabular}{llll}
\hline Cell type & Abbreviation & Axon & Dendrite \\
\hline Pyramidal cell in layer 2/3 & $\mathrm{p} 2 / 3$ & 6 & 6 \\
Basket cell in layer 2/3 & $\mathrm{b} 2 / 3$ & 6 & 4 \\
Double bouquet cell in layer 2/3 & $\mathrm{db} 2 / 3$ & 1 & 1 \\
Spiny stellate cell with axon in layer 4 & $\mathrm{ss} 4(\mathrm{~L} 4)$ & 2 & 2 \\
Spiny stellate cell with axon in layer 2/3 & $\mathrm{ss} 4(\mathrm{~L} 2 / 3)$ & 3 & 3 \\
Pyramidal cell in layer 4 & $\mathrm{p} 4$ & 3 & 3 \\
Basket cell in layer 4 & $\mathrm{~b} 4$ & 5 & 3 \\
Pyramidal cell in layer 5 with axon in layer 2 and 3 & $\mathrm{p5}(\mathrm{L} 2 / 3)$ & 1 & 1 \\
Pyramidal cell in layer 5 with axon in layer 5 and 6 & $\mathrm{p5}(\mathrm{L} 5 / 6)$ & 1 & 1 \\
Basket cell in layer 5 & $\mathrm{b} 5$ & 1 & \\
Pyramidal cell in layer 6 with axon in layer 4 & $\mathrm{p} 6(\mathrm{~L} 4)$ & 6 & 6 \\
Pyramidal cell in layer 6 with axon in layer 5 and 6 & $\mathrm{p} 6(\mathrm{~L} 5 / 6)$ & 1 & 1 \\
Thalamic afferent of type X or $Y$ & $\mathrm{X} / \mathrm{Y}$ & 3 &
\end{tabular}

Each row shows a description of the cell type (first column), its abbreviation used in this study (second column), and the number of neurons of which the axon and dendrites were reconstructed (third and fourth columns).

number of synapses per bouton. For example, the layer 6 pyramidal cell depicted in Figure 3 formed almost all of its synapses in layer 4 (5536), 37 synapses in layer 6, 112 in layer 5, and 61 in layer $2 / 3$. It formed no synapses in layer 1 . The selective innervation of cortical layers by synapses was common to all reconstructed neurons. The total number of synapses ranged between 975 and 9641 (mean \pm SD, $4422 \pm 1929$ ). Between 40 and 99\% (81 $\pm 15 \%$ ) of the synapses of individual neurons were formed in just one layer, and between 72 and 100\% (95 $\pm 5 \%)$ in two layers. This layer selectivity is one major criteria for the classification of neurons into cell types. The (average) synaptic laminar pattern of the different cell types is shown in Figure 4.

\section{Synaptic laminar patterns of spiny cell types}

Excitatory neurons of which the axons branch extensively in layer 4 are well known from anatomical studies. They include a subtype of the spiny stellate cells (Martin and Whitteridge, 1984), a subtype of the layer 6 pyramidal cells (Gilbert and Wiesel, 1979, 1989; Martin and Whitteridge, 1984; McGuire et al., 1984; Hirsch et al., 1998), and the thalamic afferents (Ferster and LeVay, 1978; Bullier and Henry, 1979; Gilbert and Wiesel, 1979, 1983; Freund et al., 1985; Humphrey et al., 1985). See Figure 2 for coronal views of these types [ss4(L4), p6(L4), and X/Y]. We found that they formed on average $85 \pm 12 \%$ [ss4(L4)], $89 \pm 10 \%$ [p6(L4)], and $85 \pm 15 \%(\mathrm{X} / \mathrm{Y})$ of their synapses in layer 4 (Fig. 4 ). Layer 4 contains two other spiny types, the spiny stellate cells with ascending axon (Martin and Whitteridge, 1984) [Fig. 2, ss4(L2/3)] and the star pyramidal cells (Martin and Whitteridge, 1984) (Fig. $2, \mathrm{p} 4)$. The reconstructed examples of both types formed only a moderate number of synapses in layer 4 and mainly innervated layer $2 / 3$ [ss4(L2/3), $67 \pm 19 \%$; p $4,48 \pm 22 \%$ ]. The axons of the remaining spiny cell types typically avoided innervating layer 4 . The pyramidal cells in layer 2/3 (Gilbert and Wiesel, 1979, 1983; Martin and Whitteridge, 1984; Kisvárday et al., 1986; Martin, 1988) (Fig. 2, p2/3) and a subtype of pyramidal cell in layer 5 (Gilbert and Wiesel, 1979; Martin and Whitteridge, 1984) [Fig. 2, p5(L2/3)] formed most synapses in layer $2 / 3[\mathrm{p} 2 / 3,78 \pm 9 \%$; p5(L2/3), 66\%] but also provided a moderate contribution to layer 5 . Although the axon of these cells cross layer 4, they hardly branch or form synapses in layer 4. Finally, there are two spiny cell types of which the axons innervate primarily the deep layers. The deep projecting pyramidal cells of layer 5 (Gabbott et al., 1987) [Fig. 2, p5(L5/6)] formed 87\% of the synapses in layer 6; for the deep projecting layer 6 pyramidal cells (Martin, 1988; Hirsch et al., 1998) [Fig. 2, p6(L5/6)], it was 91\%.

\section{Synaptic laminar patterns of smooth cell types}

Although basket cells can form ascending or descending axonal projections, by far, most of the axonal branching is in the same layer as their soma. Basket cells in layer 2/3 (Somogyi et al., 1983; Ahmed et al., 1998; Buzás et al., 2001) (Fig. 2, b2/3) formed $93 \pm$ $6 \%$ of the synapses in layer $2 / 3$. Basket cells in layer 4 (Somogyi et al., 1983; Kisvárday et al., 1985; Ahmed et al., 1997; Buzás et al., 2001) (Fig. 2, b4) formed $90 \pm 8 \%$ of their synapses in layer 4 , and the layer 5 basket cells (Kisvárday et al., 1987) (Fig. 2, b5) formed $75 \%$ of their synapses in layer 5 . In contrast, a characteristic feature of double bouquet cells is their vertical axonal arbor that innervates several layers (Somogyi and Cowey, 1981; Martin, 1988 ) (Fig. 2, db2/3). However, a recent in vitro study in cat area 17 showed that even double bouquet cells tend to form most synapses in the same layer as their soma (Tamás et al., 1997). We found that the reconstructed double bouquet cell formed most synapses in layers $2 / 3$ and 4 ( $47 \%$ each). The four reconstructed smooth neurons of layer $2 / 3$ formed an average of $6171 \pm 1964$ synapses (Tamás et al., 1997), of which $76 \pm 6 \%$ were located in layer $2 / 3$ and only $10 \pm 5 \%$ in layer $4(2 \pm 1 \%$ in layer 5 and 6 , and $8 \pm 2 \%$ in layer 1$)$. These data were included in calculating the average number of synapses (Tamás et al., 1997) per layer formed by the double bouquet cells (Fig. $4, \mathrm{db} 2 / 3$ ). Also shown is the synaptic laminar pattern of the chandelier cell of layer $2 / 3$ (axo2/3) from the anatomical studies of Somogyi et al. (1982) and Freund et al. (1983) (see Materials and Methods).

\section{Dendritic laminar patterns}

Figure 5 shows the total length of dendrite that a neuron of a given type forms in each layer. As with axonal trees, the dendritic trees of neurons are highly layer selective. Wherever possible, we used our database of reconstructed neurons (gray bars) to provide the example dendritic tree. However, for each layer, we also included pools denoted by sm1, sp1, etc. (white bars), to represent neurons that are not one of the reconstructed types. This allows for neuronal types known to exist for which the necessary data are missing (e.g., spiny neurons in layer 1). The average dendritic length of the smooth neurons in these groups was set to the average dendritic length of the reconstructed smooth neurons in the database $(4.7 \mathrm{~mm})$ (Fig. $5, \mathrm{sm} 1-\mathrm{sm} 6)$. The average dendritic length of the spiny neurons in layer 1 (sp1) was set to the average length of the dendritic trees of the reconstructed spiny neurons that have no apical dendrite $(6.9 \mathrm{~mm})$ (Fig. 5, sp1). The layer 6 pyramidal cells p6(L4) and the layer 5 pyramidal cell p5(L5/6) formed a significant amount of dendrite in more than one layer, and for both cell types, only $\sim 53 \%$ of their total dendritic length was contained in the layer of soma. However, this is the exception. All other cell types have most of the dendritic trees contained in the layer of soma, where they form $>75 \%$ of their total dendritic length.

\section{Number of neurons per cell type}

There are no direct measurements of the frequency of occurrence of many cell types. For these missing numbers, we derived estimates from several sources. The final estimates are summarized in Figure $6 \mathrm{~A}$. Our starting point was the total number of spiny and smooth neurons per cell type, for which estimates of each cortical layer are available from anatomical studies (Beaulieu and Colonnier, 1983, 1985; Gabbott and Somogyi, 1986) (see Materials and Methods). For each layer, we estimated the proportion of neurons of a given cell type and, therefore, the absolute cell numbers as well. 


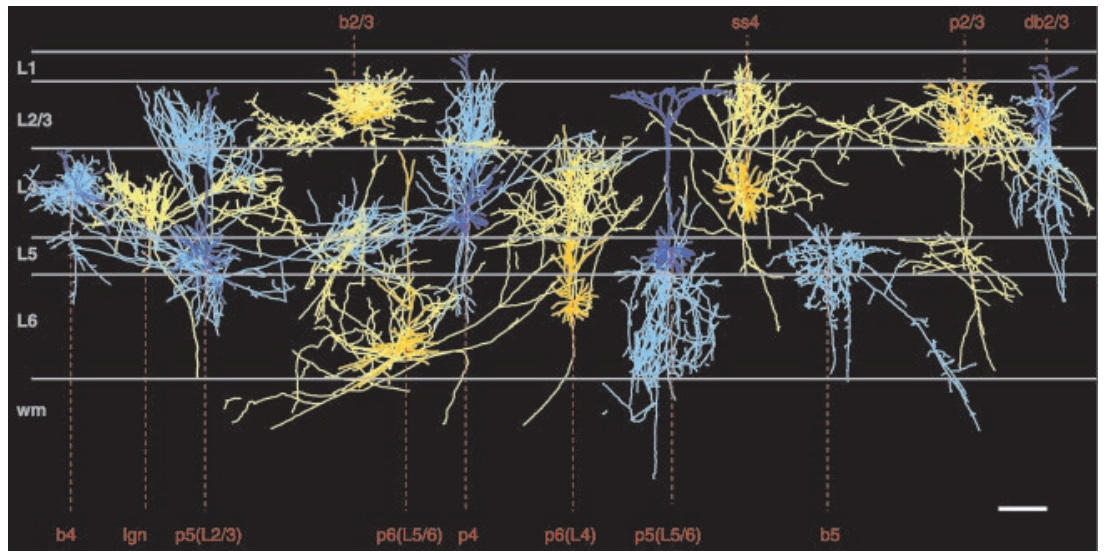

Figure 2. Coronal view of reconstructed cells representing the different cell types present in the database. For better viewing, two-color schemes (blue and yellow) were used. Axons are shown in bright blue or bright yellow, and dendrites are shown in dark blue or dark yellow. Boutons are skipped for visibility. Cell types are indicated at the top. b2/3, b4, b5, Basket cells in layer 2/3, 4, and 5; db2/3, double bouquet cell in layer 2/3; p2/3, p4, p5, p6, pyramidal cells in layer 2/3, 4, 5, and 6; ss4, spiny stellate cells in layer 4. Spiny stellate cells and pyramidal cells in layer 5 and 6 were further distinguished by the preferred layer of the axonal innervation [ss4(L4) (data not shown), ss4(L2/3), p5(L2/3), p5(L5/6), p6(L4) and p6(L5/6)]. X/Y thalamic afferents of type $X$ and Y. Horizontal lines indicate the approximate cortical layers L1, L2/3 (layer 2 and 3 were merged), L4, L5, and L6. Also indicated is the white matter (wm). Scale bar, $300 \mu \mathrm{m}$.

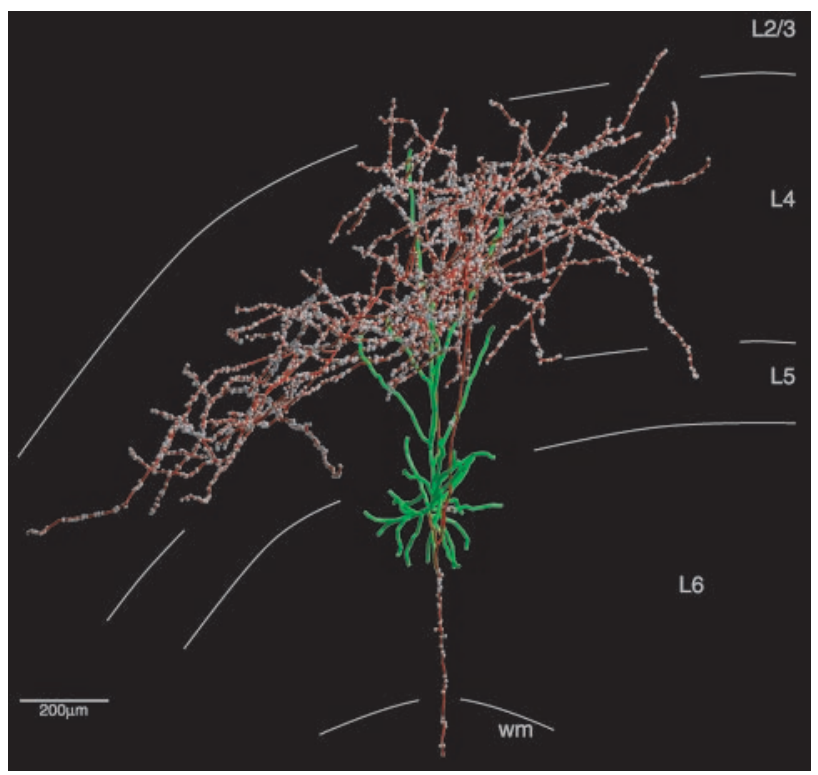

Figure 3. Coronal view of a reconstructed layer 6 pyramidal cell. The axon is shown in red, boutons are in white, and dendrites are in green. Cortical layers are indicated by gray curves. Receptive field: simple (s1), monocular driven, preferred orientation at $60^{\circ}$; size, $0.3 \times 0.5^{\circ}$. wm, White matter.

\section{Spiny cell types, including thalamic afferents}

The number of pyramidal cells in layer $2 / 3$ (p2/3) and the number of spiny cells of type sp 1 in layer 1 are known because there is no other spiny cell type present. All other layers contain at least two types of spiny neurons. For layer 4, these are the two types of spiny stellate cells [ss4(L4) and ss4(L2/3)] and the pyramidal cells of this layer (p4). Because we have no additional information about their relative frequency, we assumed that all three cell types each contribute one-third of the spiny neurons of layer 4 . There are no numerical data available for the two types of pyramidal cells in layer 5 [p5(L2/3) and p5(L5/6)]. However, pyramidal cells in layer 5 with descending axon [i.e., p5(L5/6)] tend to have large cell bodies (Martin and Whitteridge, 1984; Gabbott et al., 1987). Large cell bodies form $\sim 21 \%$ of all pyramidal cell somata in layer
5 (Peters and Yilmaz, 1993). We therefore assumed that neurons of type $\mathrm{p} 5(\mathrm{~L} 5 / 6)$ form $21 \%$ of the pyramidal cells in layer 5 , and the remaining $79 \%$ are all of type $\mathrm{p} 5(\mathrm{~L} 2 / 3)$. We estimated that $75 \%$ of the pyramidal cells in layer 6 had axons arborizing in layer 4, whereas the remaining spiny cells in this layer are the pyramidal cells with an axon restricted to layer 5 and 6 [p6(L5/6)] (Gilbert and Kelly, 1975; Martin and Whitteridge, 1984; Katz, 1987; Ahmed et al., 1994). Finally, the number of thalamic afferents of type $\mathrm{X}$ and $\mathrm{Y}$ per square millimeter of cortical area entering V1 is $\sim 902$ (Peters and Payne, 1993). By multiplying this number with the surface area of area 17 , we obtained a total of $0.36 \times 10^{6}$ thalamic afferents of type $\mathrm{X}$ and $\mathrm{Y}$.

\section{Smooth cell types}

GABAergic cortical neurons that are immunoreactive for parvalbumin (PV-IR) have the morphological features of basket cells and chandelier cells, and those GABAergic neurons immunoreactive to calbindin (CB-IR) have the morphological features of double bouquet cells, Martinotti cells, and neurogliaform cells (Alcantara and Ferrer, 1994; DeFelipe, 1997). Although chandelier cells are located primarily in layer 2/3 (Fairen and Valverde, 1980; Somogyi et al., 1982), where they form $\sim 3.4 \%$ of the GABAergic neurons (see Materials and Methods), Martinotti cells and neurogliaform cells are located principally outside layer 2/3 (Peters and Regidor, 1981; Wahle, 1993). We therefore assumed that the PV-IR neurons in layer 4 and 5 are basket cells, and the CB-IR neurons in layer $2 / 3$ are the double bouquet cells. PV-IR neurons in layer $2 / 3$ form between 37 and 54\% of the GABAergic neurons in layer 2/3 (Demeulemeester et al., 1989; Huxlin and Pasternak, 2001). By subtracting the $3.4 \%$ formed by the chandelier cells, we calculated that $\sim 42 \%$ of the GABAergic neurons in layer $2 / 3$ are basket cells. In layer 4 and 5, the proportion of GABAergic neurons that are PV-IR (and thus basket cells) is 78 and 42\%, respectively (Demeulemeester et al., 1989; Hogan et al., 1992; Huxlin and Pasternak, 2001). We estimated that the proportion of the double bouquet cells in layer $2 / 3$ is $\sim 25 \%$, because the CB-IR neurons in this layer form between 20 and $30 \%$ of the GABAergic neurons (Demeulemeester et al., 1989; Hogan et al., 1992; Huxlin and Pasternak, 2001). By definition, the number of neurons in sm1-sm 6 is given by the remaining smooth cells in layers $1-6$.

\section{"Missing" types}

In our in-depth survey of the literature, we found only a few exceptions where the description of the morphology of a cell type differed in some aspects from the reconstructed neurons used here. One example is the layer 5 pyramidal cells of which the axons innervating layer $2 / 3$ and layer 5 at approximately equal proportions also do exist (Martin and Whitteridge, 1984). We do not have three-dimensional reconstructions of these cells in our database. One study also revealed a layer 6 pyramidal cell with a dominant projection in layer 2/3 (Hirsch et al., 1998). Two of the reconstructed layer 6 pyramidal cells in our database were similar in that they also innervated the lower part of layer $2 / 3$, but the number of boutons formed in this layer was $<23 \%$. Finally, basket cells in layer 5 were observed with an ascending axonal pro- 


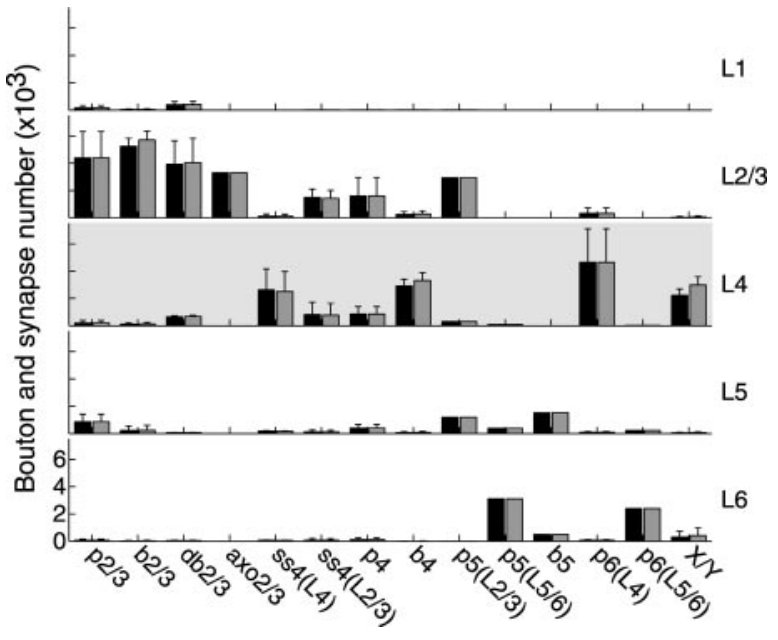

Figure 4. Synaptic laminar pattern of cell types. For each cell type (indicated along the $x$-axis), the average number of boutons (black bars) and the average number of synapses (gray bars) that the reconstructed neurons of this type form in each cortical layer (indicated along $y$-axis) are shown. The average number of synapses per layer is the product of the average number of boutons per layer and the ratio of the number of synapses per bouton for this cell type. The ratios are as follows: pyramidal cells in layer 2/3 and5, 1.0 (Kisvárday etal., 1986; Gabbottetal., 1987); spiny stellate cells in layer 4, 0.95 (Anderson et al., 1994a); basket cells in layer 2/3, 1.03 (Buhl et al., 1997); double bouquet cells in layer 2/3, 1.09 (Buhl et al., 1997); basket cells in layer 4, 1.14 (Somogyi et al., 1983). X-type thalamic afferents had a ratio of 1.27 (Freund et al., 1985), and Y-type afferents had a ratio of 1.30 (McGuire et al., 1984) in one study and 1.64 in another study (Freund et al., 1985). We used the average of the two numbers, 1.47. For the remaining cell types, no experimental data were available, and we assumed an average number of synapses per bouton of 1.0. Layer 4 is indicated by the gray background. SDs are indicated above each bar.

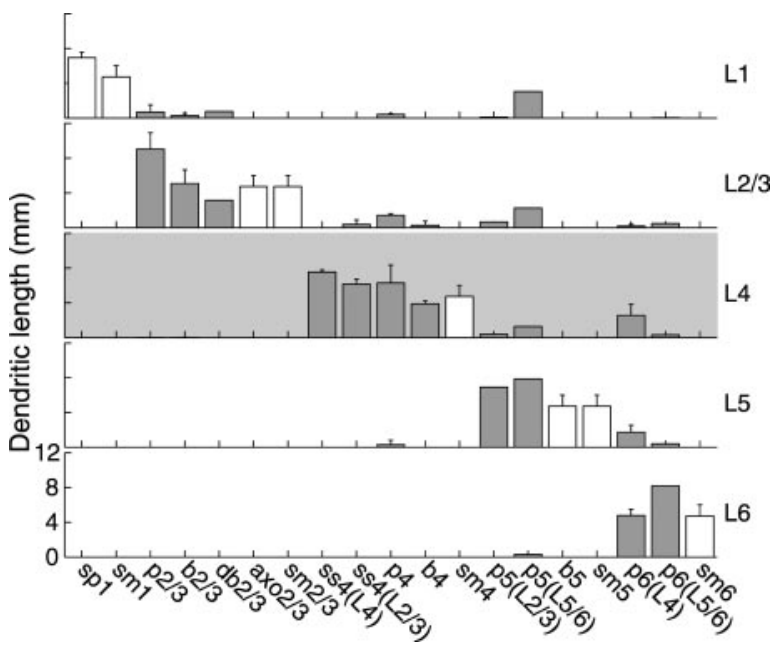

Figure 5. Dendritic laminar pattern of cell types. Gray bars indicate for each cell type (indicated along the $x$-axis) the averaged summed length of the dendritic branches that the reconstructed neurons of this type form in a each cortical layer (indicated along $y$-axis). White bars indicate estimates of the average dendritic length for the cell types not present in the database. $\mathrm{sp} 1$, Spiny cells in layer $1 ; \mathrm{sm} 1, \mathrm{sm} 2 / 3, \mathrm{sm} 4, \mathrm{sm} 5$, and $\mathrm{m} 6$ are all smooth cells in the corresponding layer that do not belong to any of the cell types $\mathrm{b} 2 / 3, \mathrm{db} 2 / 3, \mathrm{~b} 4$, and b5. SDs are indicated.

jection to layer $2 / 3$, where the axon branched again extensively (Kisvárday et al., 1987). In contrast, the reconstructed basket cell in layer 5 lacked such an ascending axon and had instead an axon descending to layer 6 .

\section{Overall statistics of synapses and dendrites}

Using stereological studies, Beaulieu and Colonnier (1985) estimated the number of symmetric and asymmetric synapses in each cortical layer of cat area 17 (see Materials and Methods). Using Figures 4 and $6 \mathrm{~A}$, we can also make estimates of these numbers. A comparison reveals (Fig. $6 B$ ) that for layers $2 / 3,4$, and 5 , the two estimates, which were derived using independent methods, are reasonably close. Exceptions are the number of synapses in layer 1 and layer 6 , for which our estimates are much lower. For each layer, we introduced a group of "unassigned" asymmetric and symmetric synapses, which account for the difference between our estimates and the estimates of Beaulieu and Colonnier. In the case where our estimates were higher, we set the number of unassigned synapses to zero.

Unassigned asymmetric synapses form only a relatively small proportion for layers $2 / 3$ and $5(<10 \%)$; however, for layer 1 , it is 93 and $32 \%$ for layer 4 and $70 \%$ for layer 6 . These synapses are probably formed by afferents of neurons located outside area 17 that are not X-type and Y-type geniculate relay cells (e.g., claustral afferents, other cortical areas). In addition, some unassigned asymmetric synapses may be attributed to the spiny neurons of layer 1, the only cortical cell type for which we do not have reconstructed samples. However, the number of spiny neurons in layer 1 is tiny ( $\sim 3 \%$ of all neurons in layer 1 ) (Beaulieu and Colonnier, 1985; Gabbott and Somogyi, 1986), and they are unlikely to account for many of the unassigned asymmetric synapses in layer 1 and layer 6.

Unassigned symmetric synapses are likely to be contributed by the various smooth neurons. We can account for almost all of the symmetric synapses in layer $2 / 3$, but $89 \%$ of the symmetric synapses in layer 1 were unassigned (36\% in layer $4,73 \%$ in layer 5 , and $96 \%$ in layer 6 ). The high number of unassigned symmetric synapses in layers 1 and 6 is simply because we had no example neurons to reconstruct. From the patterns of arborization of GABAergic neurons noted above, the unassigned symmetric synapses most likely come from neurons within the same layer.

Taking the average dendritic length of neurons of a given cell type (Fig. 5) and the total number of cells of that type (Fig. 6A), we estimated the summed length of dendritic branches that a type formed in each cortical layer (Fig. 6C). The estimated average dendritic length of the cell types not present in the database of reconstructed neurons has only a small effect on the summed length (Fig. $6 C$, white bars), because the frequency of neurons of these cell types is generally small.

\section{Number of synapses between cell types}

Using Equation 1, we are now in the position to estimate the average number of synapses the neurons of a cell type form with an individual neuron (Figs. 7, 8). In the following, we extract various aspects of these numbers.

\section{Number of synapses per neuron}

The total number of synapses per neuron was calculated by Beaulieu and Colonnier (1985) by dividing the total number of synapses by the total number of neurons. Here, we can give a more detailed account by adding the number of synapses that is formed by the different neurons of a given cell type with an individual neuron (adding the numbers in each row of Fig. 7 , including the number of unassigned synapses). This results in the number of synapses a neuron of each cell type receives on average in each layer (Fig. 9A).

The total number of synapses that a neuron receives is given by summing the synaptic input from axons in the cortical layers through which its dendrites pass (Fig. 9B). For example, the dendrites of the pyramidal cells in layer 5 of type p5(L5/6) branch extensively in layer 5 and in layer $2 / 3$ [Fig. 5, p5(L5/6)]. In layer $2 / 3$, the dendrites receive a total of 1367 synapses, and in layer 5 soma and dendrites receive a total of 4899 synapses. Also note the 
A

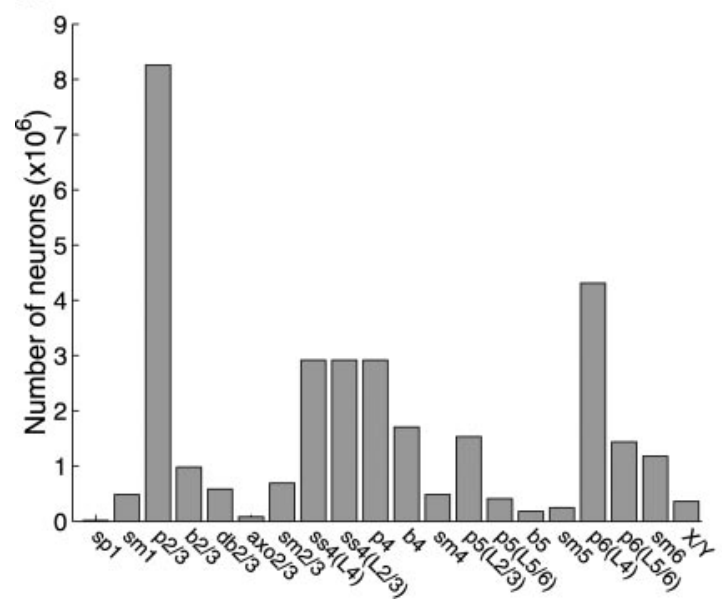

B

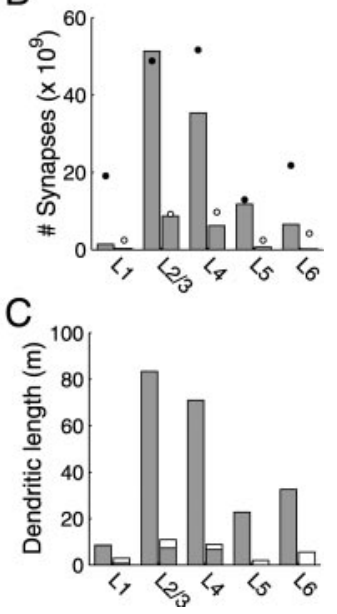

Figure 6. Estimated number of cells per cell type. $A$, Each bar indicates the estimated number of neurons per cell type ( $x$-axis). $B$, Estimated total number of synapses in each cortical layer, based on $A$ and Figure 4 . For each layer ( $x$-axis), the left bar indicates the estimated number of asymmetric synapses, and the right bar indicates the estimated symmetric synapses. Superimposed are the number of asymmetric (closed circles) and symmetric (open circles) synapses determined by Beaulieu and Colonnier (1985). C, Gray bars indicate the summed length of dendritic branches of all reconstructed cell types (left bars, spiny neurons; right bars, smooth neurons) innervating the cortical layers (indicated by the long $x$-axis). White bars on top of the gray bars indicate the summed length of dendritic branches formed by the cell types for which no example was present in the database. These cell types are indicated in Figure 5 (white bars).

high number of synapses these dendrites receive in layer 1 (5658). The reason for this is the high number of synapses in this layer and the small amount of dendrite present in layer 1. Consequently, the overall synaptic density (the number of synapses per neuron divided by the summed length of the dendritic trees of the neuron) is high for the neurons in layer 1 (188 synapses per 100 $\mu \mathrm{m})$ when compared with the neurons in the remaining layers ( $\sim 73 \pm 8$ synapses per $100 \mu \mathrm{m}$ of dendritic length) (Fig. 9 C). In general, the total number of synapses on a neuron (Fig. 9B) ranges for the different cell types between 2981 and 13075 $(5651 \pm 3120)$. The asymmetric synapses form on average $84 \pm$ $2 \%$ of the total number of synapses on the different neurons.

\section{Input to layer 4 neurons: a comparison with synaptic maps}

Using Figure 7, we calculated the proportion of synapses that the small basket cells and spiny cells in layer 4 receive from the different cortical cell types. For the calculations, we did not distinguish between the dendrites of the three different types of spiny neurons of layer 4 . We could then compare our estimates with the results of Ahmed et al. $(1994,1997)$. In their study of the synaptic maps of layer 4 spiny stellate and small basket cells, the following four sources were considered: the spiny stellate cells in layer 4 , the pyramidal cells in layer 6 with an ascending axon, the basket cells in layer 4, and the thalamic afferents (Fig. 10). They found that $45 \%$ of the asymmetric synapses formed with spiny stellate cells arose from pyramidal cells in layer 6 [p6(L4)], 28\% arose from spiny stellate cells [ss4(L4), ss4(L2/3) and p4], 6\% from thalamic afferents $(\mathrm{X} / \mathrm{Y})$, and the remainder, $21 \%$, could not be assigned to a source. Synapses belonging to basket cells formed $84 \%$ of the symmetric synapses on the dendrites. The remaining $16 \%$ of symmetric synapses could not be assigned to a source. For the small basket cells of layer 4, 90\% of the asymmetric synapses could be assigned to a presynaptic cell type: $43 \%$ of these synapses arose from pyramidal cells in layer $6,44 \%$ from spiny stellate cells, and $13 \%$ from thalamic afferents. Of the symmetric synapses, $79 \%$ were formed by basket cells in layer 4 , and the remaining $21 \%$ could not be assigned to a source. Asymmetric synapses

on dendrites of spiny stellate cells form $91 \%$ of all dendritic synapses (Anderson et al., 1994b), for the basket cells, it is 93\% (Ahmed et al., 1997). These last two proportions were used to convert the cell type-specific synaptic proportions, which relate to either asymmetric or symmetric synapses on the dendritic trees, to the total population of synapses on dendritic trees (Fig. 10, closed and open circles). Note that our estimates of the cell type-specific proportions on the spiny stellate cell and the basket cell are similar. In fact, these proportions approximate the proportion of synapses formed by the spiny stellate cells, basket cells, layer 6 pyramidal cells and thalamic afferents in layer 4 (see Eq. 3).

Compared with the experimentally derived estimates of the synaptic maps of layer 4 spiny stellates and small basket cells (Ahmed et al., 1994, 1997), the basic patterns of innervation are correctly predicted by the present method (e.g., both methods agree that the intracortical inputs from layer 6 pyramidal cells and spiny stellate cells are substantially larger than the thalamic input, with the small basket cell values lying somewhere between the thalamic and intracortical excitatory inputs). In the case of these three sources of excitatory inputs to the spiny stellate cells, the quantitative prediction is close to that seen experimentally. In the case of the small basket cells, agreement is reasonably good for the layer 6 pyramidal cells but less good for the thalamic and spiny stellate inputs (Fig. 10), although the trend is as predicted. Possible reasons for the discrepancies are suggested in Discussion.

\section{Change of synaptic maps with neuron number}

By relying on published anatomical data from other groups, we lay ourselves open to the criticism that, because these data were collected under different protocols, only relative values are reliable. We used relative values where possible, and on the occasion where absolute measurements are required, we referred to studies where corrections for tissue shrinkage was made (Beaulieu and Colonnier, 1983, 1985). This minimizes the variance caused by taking data from different studies. However, the largest uncertainty is likely to be introduced in our estimate of the proportion of neurons belonging to a given cell type, because many of these estimates are not based on direct experimental measurements. Here, we give some estimate of the consequences of changing the proportion of cell types. The bars in Figure 10 indicate the range of proportions of synapses formed with layer 4 basket cells and spiny stellate cells when the proportion of neurons of type b4, ss4(L4), and p6(L4) were changed to $\alpha_{j}+\Delta \alpha[\Delta \alpha \epsilon(-0.2,0.2)]$ while leaving the total number of excitatory and inhibitory neurons in each layer unchanged (see Materials and Methods). Here, $\alpha_{j}$ denotes the undisturbed proportion of inhibitory neurons (b4) or excitatory neurons [ss4(L4) and p6(L4)] in the corresponding layers. In general, the proportion of the total number of synapses on an arbitrary neuron formed by different presynaptic types deviates between -5.3 and $5.3 \%$ from their original values if any of the presynaptic cell types is perturbed by $\Delta \alpha=0.1$. For $\Delta \alpha=0.2$, the range is between -10.5 and $10.5 \%$ (i.e., the range of differences scales linearly with $\Delta \alpha$ ).

Based on theoretical estimates, Budd (2000) found that layer 4 basket cells form between 193 and 365 synapses with spiny neu- 

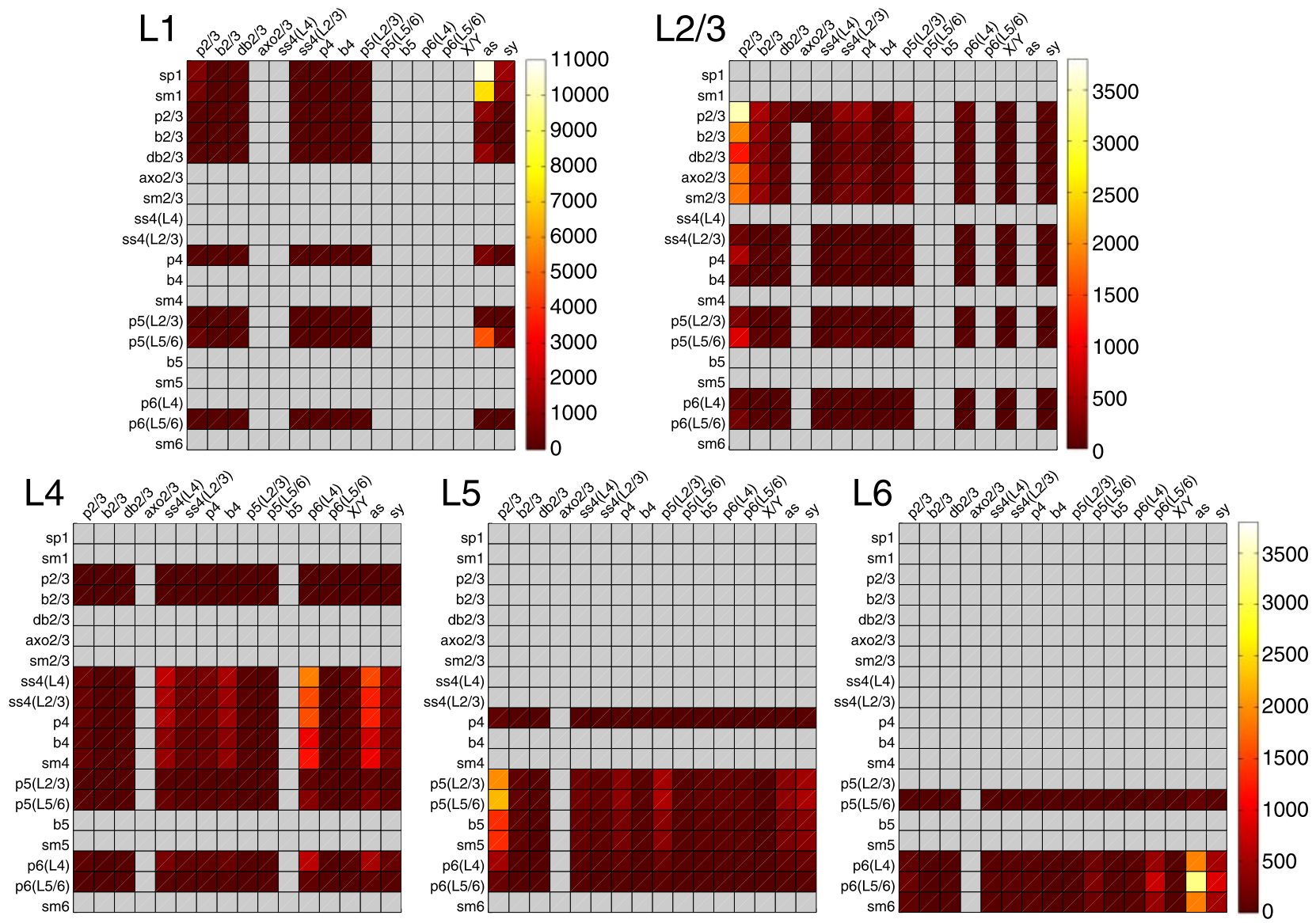

Figure 7. Estimated number of synapses formed by one type of neuron with another type in the cortical layer indicated based on Peters's rule (Eqs. 2,3). L1-L6, Presynaptic cell types are indicated on the top, postsynaptic cell types are indicated along the $y$-axis. For a presynaptic cell type $j$ and a postsynaptic cell type $i$, the color of the corresponding square indicates the number of synapses that all neurons of type $j$ form in layer $u$ (indicated on the top left corner) with an individual neuron of type $i$. This number is denoted by $\bar{s}_{i j}^{u}$ in Figure 1. Light gray squares indicate zero values. sy and as indicate the number of unassigned asymmetric and symmetric synapses on the dendritic trees of each cell type. Color bars are shown.

rons in layer 4, and between 75 and 159 synapses with smooth neurons in layer 4. This is lower than our estimates of 486 and 348 synapses, respectively. A major difference to our approach is Budd's assumption of a significantly lower proportion of layer 4 basket cells ( $26-46 \%$ of all layer 4 inhibitory neurons, compared with our estimate of 78\%). Using a lower proportion of layer 4 basket cells (i.e., $40 \%$ ), we get similar synapse numbers (248 synapses onto spiny neurons and 178 synapses onto smooth neurons).

\section{Anatomical weight of the projections}

Finally, with these estimates, we can now describe the quantitative circuit for cat area 17. Figure $11 A$ shows the total number of synapses that the different cell types form with an individual neuron. The mean number of synapses arising from pools of one type of cell is $260 \pm 493$ (unassigned synapses excluded). It is clear that there is strong layer selectivity of axonal and dendritic trees (Figs. 4, 5). Either dendritic trees and axonal trees overlap strongly within a cortical layer, with the consequence that the postsynaptic dendritic trees receive a large number of synapses from the presynaptic cell types, or the overlap is only modest, and hardly any synapses are made between presynaptic and postsynaptic elements (Fig. 7, L2/3, rows $\mathrm{p} 2 / 3, \mathrm{~b} 2 / 3, \mathrm{db} 2 / 3$, and $\mathrm{sm} 2 / 3$ ). The histogram of the number of synapses that are formed between cell types and individual neurons (Fig. $11 \mathrm{~B}$ ) shows that for many of these, the synaptic numbers are rather low (i.e., "weak projections") and only a few are very high (i.e., "strong projections").

\section{Strong projections}

The synaptic input of most neurons in layer $2 / 3$ and 5 is dominated by one major source, the layer $2 / 3$ pyramidal cells, which form between 40 and $60 \%$ of all synapses on the dendritic trees and between four and six times more synapses than the next dominant cell type (Fig. 11A,C). The only exceptions are the double bouquet cells in layer $2 / 3$ and one type of layer 5 pyramidal cell [type p5(L5/6)], because their dendrites extend to layer 1 where there are many synapses. Each neuron in layer $2 / 3$ receives on average 2094 synapses ( \pm 857 ; range, $1293-3554$ ) from other layer $2 / 3$ pyramidal cells. Each layer 5 neuron receives on average 2121 synapses ( \pm 1018 ; range, $1357-3508$ ) from the layer $2 / 3$ pyramidal cells. For comparison, layer 6 pyramidal cells, which provide the dominant input to layer 4, form on average 1443 synapses with each layer 4 neuron ( \pm 361 ; range, 980-1895), which is between 20 and $40 \%$ of the total number of synapses made with each layer 4 neuron.

\section{Weak projections}

In addition to these dominant projections, cortical neurons always received a small number of synapses from many different cell types. For each neuron of a given postsynaptic cell type, the number of cell types forming between 5 and 300 synapses with a neuron (which is $<10 \%$ of the total number of synapses on the dendritic tree) ranges between 4 and 11 (of a total of 14 presynaptic cell types). These weak projections form on average $98 \pm 78$ synapses with a target neuron. 


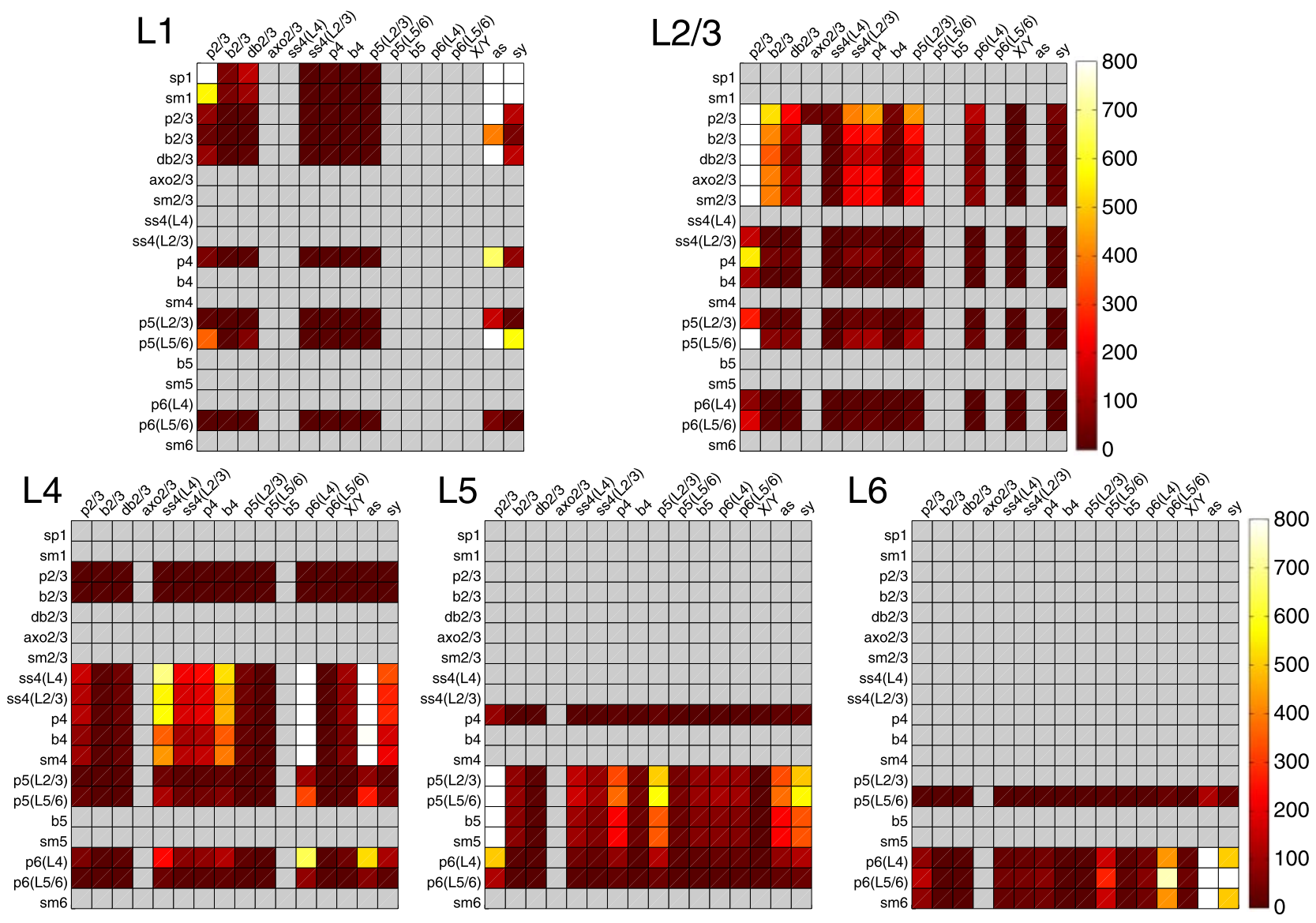

Figure 8. Same as for Figure 7, but only the weak projections (those forming $<800$ synapses) are color coded. The stronger projections are shown in white.
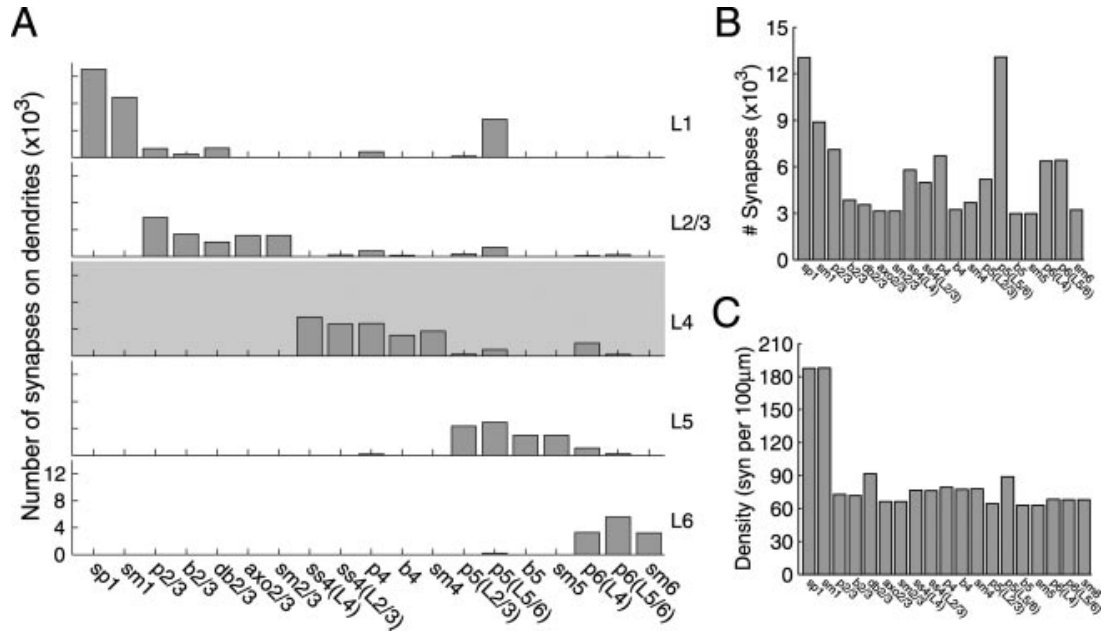

Figure 9. Number of synapses per neuron. A, Average number of synapses formed with neurons, calculated from Figure 7. Each bar indicates the number of synapses that a neuron of a given cell type (indicated along the $x$-axis) receives in each cortical layer (indicated along the $y$-axis). B, Summing over the cortical layers in $A$ results in the total number of synapses a neuron of a given cell type ( $x$-axis) receives. C, Dividing this number by the summed length of the dendritic branches of the dendritic trees results in the overall synaptic density of synapses along the dendritic trees of cortical cell types.

\section{Discussion}

Decades of careful work have been devoted to establishing the quantitative anatomy of the cat area 17. In no other species or cortical area are there as complete quantitative descriptions of the neuronal types, dendrites, axons, and synapses as those that exist for cat area 17 . We used these data together with our own library of reconstructed neurons to make a quantitative estimate of the total local excitatory circuit. Our results show that by applying an elaboration of the simple connectivity rule of Peters (Braitenberg and Schüz, 1991), a complex network structure is generated. A great advantage of this approach is that this rule produces an objective and quantitative model of cortical connectivity that incorporates the major cell types and the thalamic afferents. Furthermore, as more detailed data about the connections made by subtypes of neurons are accrued, the new data can be easily incorporated in the model. Finally, the predictions are in principle testable, as we have illustrated by comparing our estimates with the mapped input to the layer 4 spiny stellate and small basket cells (Ahmed et al., 1994, 1997).

We were able to show good quantitative agreement for the two methods in the case of the input to the spiny stellate cells. In the case of the small basket cells, the quantitative agreement was good for the layer 6 pyramidal cells, but the agreement was only qualitative in the case of the other inputs. Part of this discrepancy between the two estimates may be because in their experimental study, Ahmed et al. (1997) found evidence for two additional excitatory inputs and at least one 


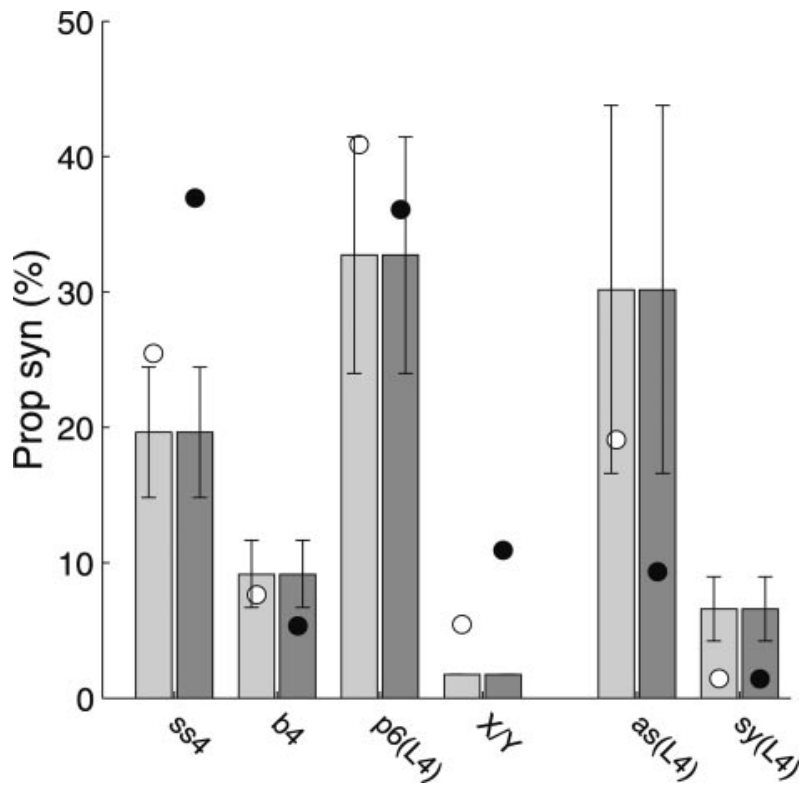

Figure 10. Proportion of synapses (Prop syn) on layer 4 neurons. Bars indicate our estimate of the proportion of synapses that the cell types (indicated along the $x$-axis) form with a spiny stellate cell (light shaded bars) and a basket cell (dark shaded bars) in layer 4. The estimates are based on Figure 7. as $(L 4)$ and sy $(L 4)$ indicate the proportion of unassigned asymmetric and symmetric synapses. ss 4 indicates the cell types $p 4$, ss4(L4), and ss $4(\mathrm{~L} 2 / 3)$, which we pooled for the calculations. Error bars indicate the variability in the proportions due to variations in cell number (see Results). Each circle indicates the proportion of synapses that the cell types formed with a spiny stellate cell (light shaded circles) and a basket cell (dark shaded circles). Data are based on the studies by Ahmed et al. $(1994,1997)$.

inhibitory input to the small basket cells, but they could not identify the sources. The "unknown" asymmetric synapses were formed by medium-sized boutons, so they did not originate from layer 6 pyramidal cells, which form small boutons. If the source was known, these medium sized boutons would most likely dilute somewhat the estimated contribution of the spiny stellate cells. For the symmetric synapses, Ahmed et al. (1997) estimated the upper limit for the proximal dendritic input from other small basket cells at $79 \%$, thus leaving a minimum of $21 \%$ of the symmetric synapses unassigned. It remains for experiments to discover whether resolving these uncertainties would lead to more correspondence between the theoretical and experimental estimates. However, one benefit of the present method is that it identifies possible other sources of asymmetric and symmetric synapses to spiny stellate and small basket cells that could not be identified in the original experimental studies (Ahmed et al., 1994, 1997).

There are only a few other studies that have attempted to model the number of synapses formed between neurons of different cortical cell types (Braitenberg and Lauria, 1960; Krone et al., 1986; Thomas et al., 1991), and these had to rely on incomplete and qualitative morphological data derived from Golgistained neurons. However, for those models, as with the present one, the resulting weighing of the nodes in the circuit depends crucially on the fidelity of the morphological reconstructions, which is therefore a key parameter. We were able to improve previous estimates by using accurate and quantitative morphological characterizations of single neurons (Figs. 4, 5). The other crucial parameter of the connectivity model is the number of cells per cell type, and these data were obtained from an intensive review of the literature (Fig. 6A).

\section{Synaptic weights}

One neglected consideration in most circuit diagrams and their functional interpretation is the absolute number of cells of a particular type that contribute their synapses to a given projection. An individual's axonal arbor may look impressive, but unless the number of neurons of that type is known, the anatomical weight of the projections cannot be properly assessed. Once the weighting is factored in, the results can be counter-intuitive (e.g., in the Introduction, we pointed to the calculation that the major drive to the cat's visual cortex comes from a projection that forms a mere $5 \%$ of the excitatory synapses in layer 4 ). This is not because the cortical arbors of the axons of thalamic relay cells are small; on the contrary, they are among the most impressive that we have reconstructed. The number of thalamocortical synapses is small primarily because there are only $\sim 350,000$ relay neurons to supply the whole primary visual cortex in the cat (Peters and Payne, 1993). Of course the question of the relative efficacy of the synapses of such minority pathways must also be addressed. In the case of cat thalamocortical synapses, the answer is clear: they do indeed have interesting functional properties, especially their extremely low quantal variance, but their quantal amplitudes are not greatly different from those of the spiny stellate cells they innervate (Stratford et al., 1996; Gil et al., 1999; Bannister et al., 2002).

\section{Sources of the missing synapses}

In calculating the total synaptic balance for cat area 17, we found that we could not account for all the synapses that should be there according to stereological sampling. Our calculations thus reveal the existence of "dark matter" of the cortex (i.e., synapses of which the source is unknown). Of course, likely sources are known (e.g., smooth neurons were not recovered in all laminas in our recordings, and this accounts for the large percentages of unassigned symmetric synapses in those layers). Because the dendritic structure of smooth neurons is relatively similar across types and restricted primarily to single layers, we probably have a reasonable estimate of their contribution to the dendritic targets in the neuropil. For their axons, the situation is different, for even where we have reasonable samples (e.g., for layer 4) we have probably not encountered all known smooth types despite years of recording. Their absence from our sample leaves one of the more frustrating lacuna in our quantitative analysis. Slice recordings would seem one solution to this, but in the cat, slice recordings remain rare and in general they suffer from the problem that unknown amounts of axon are cut and lost in preparation. Nevertheless, our results indicate how essential it is to obtain a more complete quantitative account of the smooth neuron population in the cat.

For the proportion of unassigned asymmetric synapses in layer 1 and 6, there are several explanations: (1) there are missing pathways yet to be identified, (2) we underestimated the average synaptic contribution or the proportion of the cells that do project to these layers, and (3) less likely, the stereological counts (Beaulieu and Colonnier, 1985) are inaccurate.

Layer 1 excitatory neurons would have to form $1.2 \times 10^{6}$ asymmetric synapses to make up the deficit in layer 1 . Similarly, each pyramidal cell in layer $2 / 3$ would have to form an additional 2140 synapses in layer 1 to account for the missing asymmetric synapses. The only other local source of asymmetric synapses to layer 1 is the layer 5 pyramidal cells (Martin and Whitteridge, 1984), which are relatively few in number. Sources outside area 17 are the afferents of the extrastriate cortical areas and subcortical areas (e.g., the W-type thalamic afferents). The thalamic input to layer 1 is likely to be small. However, layer 1 is a major target of the intercortical feedback pathways, and the contribution of these pathways could be substantial. 

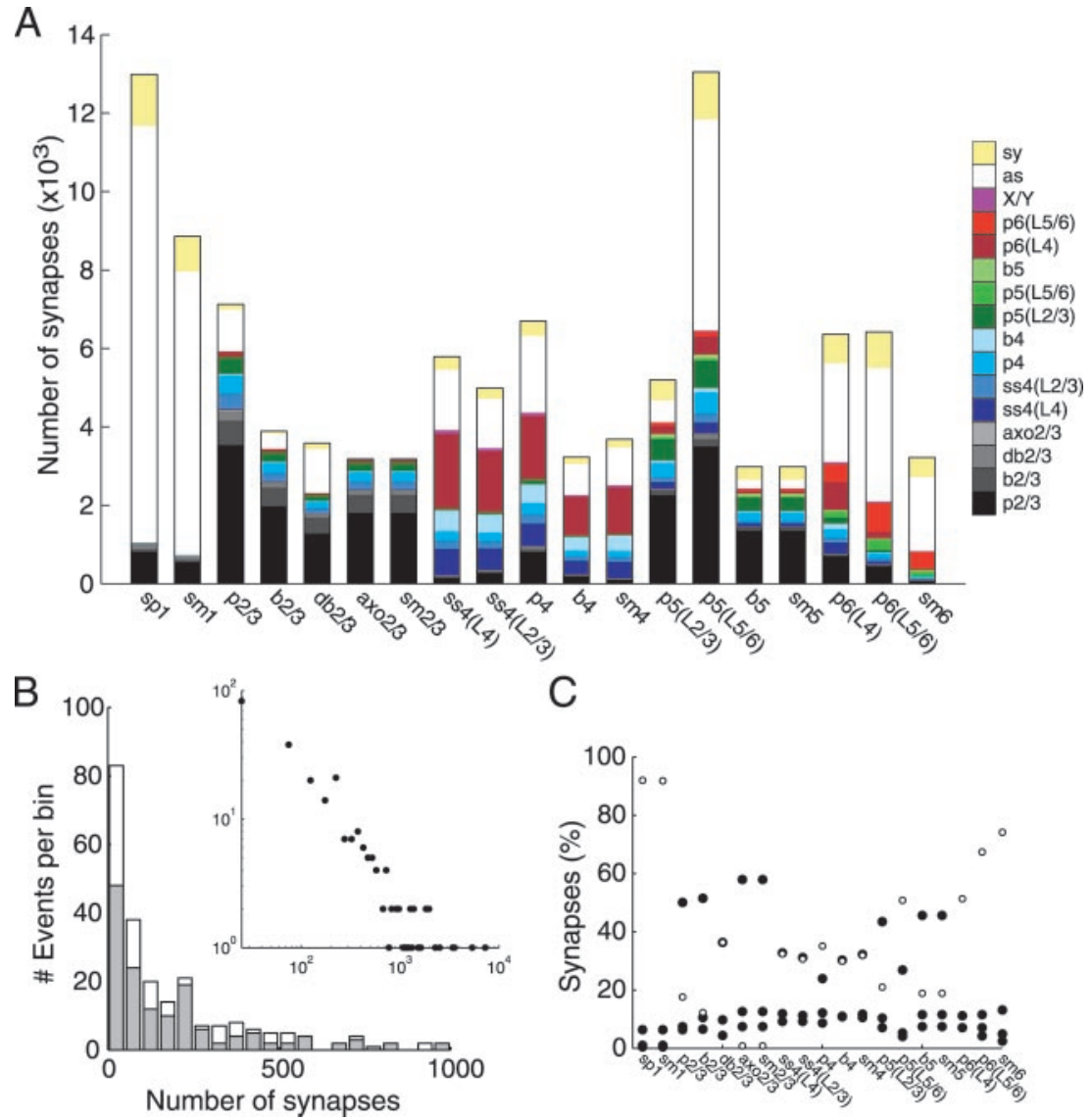

Figure 11. Number of synapses formed by the different cell types with an individual neuron. $A$, Each total bar indicates the number of synapses formed with a neuron of a given cell type (indicated along the $x$-axis). The width of each color-coded subdivision of the bar indicates the total number of synapses that a particular presynaptic cell type forms with the neurons. The correspondence between the colors and the presynaptic cell types is indicated. $B$, Histogram of synapses formed by the different cell types with an individual neuron, as shown in $A$. For visibility, projections forming $<1000$ synapses are shown only. Gray bars indicate the histogram of asymmetric synapses. Bin size is 50 synapses. Inset shows the histogram of synapses formed by all projections (log-log scale). C, For each postsynaptic cell type ( $x$-axis), the three largest proportions of synapses of its dendritic trees that were formed by the different cell types are shown (closed circles). Also shown for each cell type is the proportion of unassigned synapses on its dendritic trees (open circles).

Similarly, the intracortical feedback pathways also target deep layers and thus could also be a major source of the missing synapses in layer 6 .

\section{Neuronal specificity}

Our circuit describes the average connectivity between neuronal types. Thus, whether the synapse is formed specifically on a spine or dendritic shaft does not affect our calculation. However, the calculations do depend crucially on the relative vertical position of the target dendrites and the axon of the presynaptic cell type. For example, the star pyramid of layer 4, by virtue of its extended apical and basal dendrites, will form synapses with axons in the superficial layers to which the dendrites of the spiny stellate do not have access. Such an appeal to anatomy could account for differences in functional input specificity to pyramidal cells and spiny stellate cells in layer 4 of the monkey visual cortex (Yabuta and Callaway, 1998; Yabuta et al., 2001).

Unless the dendrites are deliberately restricted to a laminar compartment (Katz et al., 1989; Petersen and Sakmann, 2000), changes in the vertical position of a neuron within a layer will inevitably result in some neurons with dendrites crossing a laminar boundary, whereas others have their dendrites completely contained in one layer. This leads to differences in the populations of synapses from which the postsynaptic neurons lying in the same layer can sample (Lund et al., 1995). However, these more subtle effects are not captured in our model, because the prototypical synaptic and dendritic laminar patterns of the cell types were formed by averaging over individual neurons regardless of their vertical position within the layer.

Our calculations of connectivity considered the special case of the chandelier cells, which consistently ignore the axons of smooth GABAergic neurons. There is some indication that type-selective innervation is also possible for other groups of neurons, which future models of connectivity should incorporate once sufficient quantitative data are available. For example, anatomical studies in the rat and monkey visual cortex show that superficial calretinin-immunoreactive inhibitory neurons tend to ignore superficial excitatory neurons and primarily make synaptic contact with superficial inhibitory neurons (Meskenaite, 1997; Gonchar and Burkhalter, 1999). Selective innervation of inhibitory neurons was also found on a functional level (Gibson et al., 1999; Dantzker and Callaway, 2000), but it remains to be shown whether it originates from a particular anatomical organization of synapses.

\section{Cortical circuitry}

Although the wiring rule was chosen to be one of the simplest possible, the resulting distribution of synapses on the dendritic trees appears to be rather complex (Figs. 7, $8,11)$. From the 14 presynaptic cell types considered here, each neuron receives synapses on average from $12 \pm 2$ different cell types. Only one or two of these cell types form the majority of synapses on the target neuron. Although the number of synapses formed by the remaining cell types is low, we cannot of course assume they are functionally negligible, as the example of the thalamic projection shows. The cell types that provide the majority of synapses vary greatly with the layer where the postsynaptic neuron is located. The resulting network of projections between cell types therefore consists of a few anatomically strong projections involving many synapses and many anatomically weak projections involving only a few synapses. A systematic analysis of this structure and the functional implications is in progress.

The estimated number of cell type-specific synapses on the neurons (Figs. 7, 8, 11) imply a network of excitatory and inhibitory pathways between the different cortical layers. For the excitatory pathways taken by a visual signal arriving at cat area 17, several such networks were proposed for the cat (Szentagothai, 1978; Gilbert and Wiesel, 1981; Gilbert, 1983). The network shown here (Fig. 12A) gives the first realistic estimates of the number of synapses associated with each path. Traditionally, the big feedforward loop starting in layer 4 , which is the major recipient of thalamic afferent synapses, was emphasized. The loop leads to an excitatory signal propagating through layers $2 / 3,5$, and 6 , with a recurrent input back to layer 4 . Although this loop also is present in our map of the network, our estimates indicate 


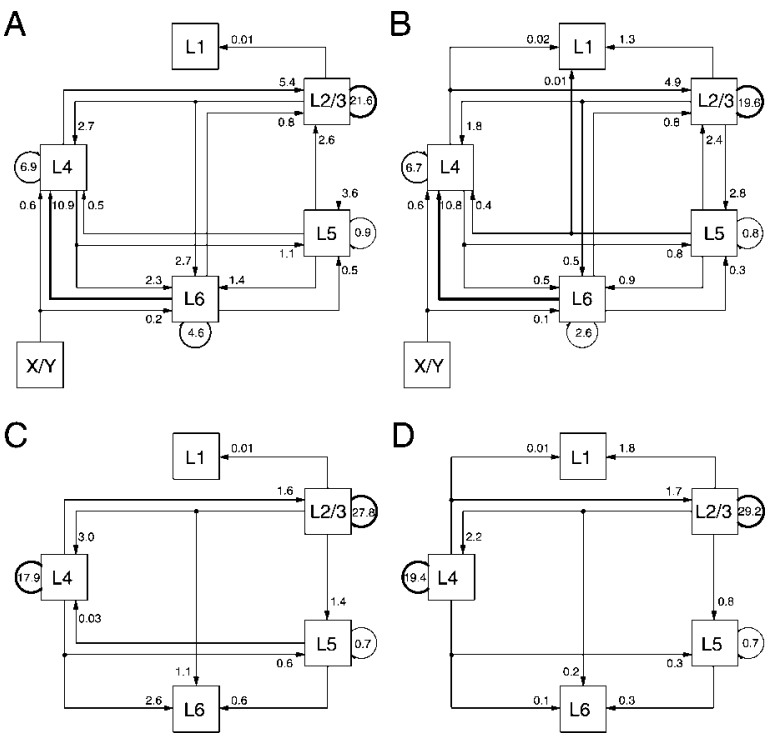

Figure 12. Number of synapses involved in the projections between excitatory and inhibitory neurons between layers, including the X-type and Y-type afferents from the dorsal LGN. The numbers were calculated based on Figure 7. $A-D$, Each arrow is labeled with a number indicating the proportion of all the synapses in area 17 that are formed between excitatory neurons $(A)$, from excitatory onto inhibitory neurons $(B)$, from inhibitory onto excitatory neurons $(C$, and between inhibitory neurons $(D)$. $A$, Total number of synapses between excitatory neurons is $13.6 \times 10^{10}$. The proportion of asymmetric unassigned synapses that the excitatory neurons in each layer receive is $0.1 \%$ (layer 1 ), 6\% (layer 2/3), 10\% (layer 4), 2\% (layer 5), and 12\% (layer 6). These synapses are presumably formed by the afferents originating outside area 17. B, Total number of synapses from excitatory neurons onto inhibitory neurons is $2.1 \times 10^{10}$. The proportion of asymmetric unassigned synapses that the inhibitoryneurons in each layer receive is 17\% (layer 1), 5\% (layer 2/3), 9\% (layer 4), $0.5 \%$ (layer 5), and 11\% (layer 6).C, Total number of synapses from inhibitory neurons onto excitatory neurons is $2.4 \times 10^{10}$. The proportion of symmetric unassigned synapses that the excitatory neurons in each layer receive is $0.1 \%$ (layer 1), 6\% (layer 2/3), 12\% (layer 4), 6\% (layer 5), and 19\% (layer 6).D, Total number of synapses between inhibitory neurons is $0.4 \times 10^{10}$. The proportion of symmetric unassigned synapses that the inhibitory neurons in each layer receive is $11 \%$ (layer 1), $5 \%$ (layer 2/3), 10\% (layer 4), 4\% (layer 5), and 15\% (layer 6).

that the total number of synapses associated with this loop is only $\sim 21 \%$ of all asymmetric synapses in area 17 that are formed between excitatory neurons. At least as many synapses (34\%) are involved in the self-innervation of the individual cortical layers, which are mediated by axons running horizontally within a cortical layer. Self-innervation of a layer is also the prominent feature of the pathways connecting inhibitory neurons and inhibitory and excitatory neurons (Fig. $12 \mathrm{~B}-\mathrm{D}$ ). An estimation of the number of synapses formed between horizontally displaced neurons is, therefore, an important next step in the quantitative characterization of cortical circuitry.

\section{References}

Ahmed B, Anderson JC, Douglas RJ, Martin KAC, Nelson JC (1994) Polyneuronal innervation of spiny stellate neurons in cat visual cortex. J Comp Neurol 341:39-49.

Ahmed B, Anderson JC, Martin KAC, Nelson JC (1997) Map of the synapses onto layer 4 basket cells of the primary visual cortex of the cat. J Comp Neurol 380:230-242.

Ahmed B, Anderson JC, Douglas RJ, Martin KAC, Whitteridge D (1998) Estimates of the net excitatory currents evoked by visual stimulation of identified neurons in cat visual cortex. Cereb Cortex 8:462-476.

Alcantara S, Ferrer I (1994) Postnatal development of parvalbumin immunoreactivity in the cerebral cortex of the cat. J Comp Neurol 348:133-149.

Anderson JC, Douglas RJ, Martin KAC, Nelson JC (1994a) Synaptic output of physiologically identified spiny stellate neurons in cat visual cortex. J Comp Neurol 341:16-24.

Anderson JC, Douglas RJ, Martin KAC, Nelson JC (1994b) Map of the syn- apses formed with the dendrites of spiny stellate neurons of cat visual cortex. J Comp Neurol 341:25-38.

Anderson PA, Olavarria J, Sluyters RCV (1988) The overall pattern of ocular dominance bands in cat visual cortex. J Neurosci 8:2183-2200.

Bannister NJ, Nelson JC, Jack JJ (2002) Excitatory inputs to spiny cells in layers 4 and 6 of cat striate cortex. Philos Trans R Soc Lond B Biol Sci 357:1793-1808.

Beaulieu C, Colonnier M (1983) The number of neurons in the different laminae of the binocular and monocular regions of area 17 in the cat. J Comp Neurol 217:337-344.

Beaulieu C, Colonnier M (1985) A laminar analysis of the number of roundasymmetric and flat-symmetric synapses on spines, dendritic trunks, and cell bodies in area 17 of the cat. J Comp Neurol 231:180-189.

Bolz J, Gilbert CD (1986) Generation of end-inhibition in the visual cortex via interlaminar connections. Nature 320:362-365.

Bolz J, Gilbert CD, Wiesel TN (1989) Pharmacological analysis of cortical circuitry. Trends Neurosci 12:292-296.

Botha D, Douglas RJ, Martin KAC (1987) TRAKA: a microcomputerassisted system for digitizing the three-dimensional structure of neurones. J Physiol (Lond) 394:16P.

Braitenberg V, Lauria F (1960) Toward a mathematical description of the grey substance of nervous systems. Nuovo Cimento 18:1135-1151.

Braitenberg V, Schüz A (1991) Peters' Rule and White's Exceptions. In: Anatomy of the cortex, pp 109-112. Berlin: Springer.

Budd JM (2000) Inhibitory basket cell synaptic input to layer IV simple cells in cat striate visual cortex (area 17): a quantitative analysis of connectivity. Vis Neurosci 17:331-343.

Buhl EH, Tamás G, Szilagyi T, Stricker C, Paulsen O, Somogyi P (1997) Effect, number and location of synapses made by single pyramidal cells onto aspiny interneurones of cat visual cortex. J Physiol (Lond) 500:689-713.

Bullier J, Henry G (1979) Laminar distribution of first order neurons and afferent terminals in cat striate cortex. J Neurophysiol 42:1271-1281.

Buzás P, Eysel UT, Adorján P, Kisvárday Z (2001) Axonal topography of cortical basket cells in relation to orientation, direction, and ocular dominance maps. J Comp Neurol 437:259-285.

Dantzker JL, Callaway EM (2000) Laminar sources of synaptic input to cortical inhibitory interneurons and pyramidal neurons. Nat Neurosci 3:701-707.

DeFelipe J (1997) Types of neurons, synaptic connections and chemical characteristics of cells immunoreactive for calbindin-D28K, parvalbumin and calretinin in the neocortex. J Chem Neuroanat 14:1-19.

Demeulemeester H, Vandesande F, Orban GA, Heizmann CW, Pochetx R (1989) Calbindin D-28K and parvalbumin immunoreactivity is confined to two separate neuronal subpopulations in the cat visual cortex, whereas partial coexistence is shown in the dorsal lateral geniculate nucleus. Neurosci Lett 99:6-11.

Douglas RJ, Martin KAC, Whitteridge D (1991) An intracellular analysis of the visual responses of neurons in cat visual cortex. J Physiol (Lond) 440:659-696.

Fairen A, Valverde F (1980) A specialized type of neuron in the visual cortex of cat. A golgi and electron microscope study of chandelier cells. J Comp Neurol 194:761-779.

Farinas I, DeFelipe J (1991) Patterns of synaptic input on corticocortical and corticothalamic cells in the cat visual cortex. II. The axon initial segment. J Comp Neurol 304:70-77.

Ferster D, LeVay S (1978) The axonal arborizations of lateral geniculate neurons in the striate cortex of the cat. J Comp Neurol 182:923-944.

Freund TF, Martin KAC, Smith AD, Somogyi P (1983) Glutamate decarboxylaseimmunoreactive terminals of Golgi-impregnated axo-axonic cells of presumed basket cells in synaptic contact with pyramidal neurons of the cat's visual cortex. J Comp Neurol 221:263-278.

Freund TF, Martin KAC, Whitteridge D (1985) Innervation of cat visual areas 17 and 18 by physiologically identified X- and Y-type thalamic afferents. I. Arborization patterns and quantitative distribution of postsynaptic elements. J Comp Neurol 342:263-274.

Freund TF, Maglóczky Z, Soltesz I, Somogyi P (1986) Synaptic connections, axonal and dendritic patterns of neurons immunoreactive for cholecystokinin in the visual cortex of the cat. Neuroscience 19:1133-1159.

Friedlander MJ, Stanford LR (1984) Effects of monocular deprivation on the distribution of cell types in the $\mathrm{LGN}_{d}$ : a sampling study with finetipped micropipettes. Exp Brain Res 53:451-461.

Gabbott PL, Somogyi P (1986) Quantitative distribution of GABA- 
immunoreactive neurons in the visual cortex (area 17) of the cat. Exp Brain Res 61:323-331.

Gabbott PLA, Martin KAC, Whitteridge D (1987) Connections between pyramidal neurons in layer 5 of cat visual cortex (area 17). J Comp Neurol 259:364-381.

Garey LJ, Powell TP (1971) An experimental study of the termination of the lateral geniculo-cortical pathway in the cat and monkey. Proc R Soc Lond B Biol Sci 179:41-63.

Gibson JR, Beierlein M, Connors B (1999) Two networks of electrically coupled inhibitory neurons in neocortex. Nature 402:75-79.

Gil Z, Connors BW, Amitai Y (1999) Efficacy of thalamocortical and intracortical synaptic connections: quanta, innervation, and reliability. Neuron 23:385-397.

Gilbert CD (1983) Microcircuitry of the visual cortex. Annu Rev Neurosci 6:217-247.

Gilbert CD, Kelly JP (1975) The projections of cells in different layers of the cat's visual cortex. J Comp Neurol 163:81-106.

Gilbert CD, Wiesel TN (1979) Morphology and intracortical projections of functionally characterized neurons in cat visual cortex. Nature 280:120-125.

Gilbert CD, Wiesel TN (1981) Laminar specialization and intracortical connections in cat primary visual cortex. In: The organization of the cerebral cortex (Schmitt FO, Worden FG, Adelman G, Dennis SG, eds), pp 163 191. Cambridge, MA: MIT.

Gilbert CD, Wiesel TN (1983) Clustered intrinsic connections in cat visual cortex. J Neurosci 3:1116-1133.

Gilbert CD, Wiesel TN (1989) Columnar specificity of intrinsic horizontal and corticocortical connections in cat visual cortex. J Neurosci 9:2432-2442.

Gonchar Y, Burkhalter A (1999) Connectivity of GABAergic calretininimmunoreactive neurons in rat primary visual cortex. Cereb Cortex 9:683-696.

Henry GH, Harvey AR, Lund JS (1979) The afferent connections and laminar distribution of cells in the cat striate cortex. J Comp Neurol 187:725-744.

Hirsch JA, Gallagher CA, Alonso JM, Martinez LM (1998) Ascending projections of simple and complex cells in layer 6 of the cat striate cortex. J Neurosci 18:8086-8094.

Hogan D, Terwilleger ER, Berman NEJ (1992) Development of subpopulations of GABAergic neurons in cat visual cortical areas. NeuroReport 3:1069-1072.

Hubel DH, Wiesel TN (1962) Receptive fields, binocular interaction and functional architecture in the cat's visual cortex. J Physiol (Lond) 160:106-154.

Hubel DH, Wiesel TN (1965) Receptive fields and functional architecture in two nonstriate visual areas (18 and 19) of the cat. J Neurophysiol 28:229-289.

Humphrey AL, Sur M, Uhlrich DJ, Sherman SM (1985) Termination patterns of individual $\mathrm{X}$ - and $\mathrm{Y}$-cell axons in the visual cortex of the cat: projections to area 18 , to the $17 / 18$ border region, and to both areas 17 and 18. J Comp Neurol 233:190-212.

Huxlin K, Pasternak T (2001) Long-term neurochemical changes after visual cortical lesions in the adult cat. J Comp Neurol 429:221-241.

Katz LC (1987) Local circuitry of identified projection neurons in cat visual cortex brain slices. J Neurosci 7:1223-1249.

Katz LC, Gilbert CD, Wiesel TN (1989) Local circuits and ocular dominance columns in monkey striate cortex. J Neurosci 9:1389-1399.

Kisvárday ZF, Martin KAC, Whitteridge D, Somogyi P (1985) Synaptic connections of intracellularly filled clutch neurons: a type of small basket neuron in the visual cortex of the cat. J Comp Neurol 241:111-137.

Kisvárday ZF, Martin KAC, Freund TF, Maglóczky ZS, Whitteridge D, Somogyi P (1986) Synaptic targets of HRP-filled layer III pyramidal cells in the cat striate cortex. Exp Brain Res 64:541-552.

Kisvárday ZF, Martin KAC, Friedlander MJ, Somogyi P (1987) Evidence for interlaminar inhibitory circuits in striate cortex of cat. J Comp Neurol 260:1-19.

Krone G, Mallot H, Palm G, Schuz A (1986) Spatiotemporal receptive fields: a dynamical model derived from cortical architectonics. Proc R Soc Lond B Biol Sci 226:421-444.

Lorente de Nó R (1949) Cerebral cortex: architecture, intracortical connections, motor projections. In: Physiology of the nervous system (Fulton J, ed), pp 288-313. New York: Oxford UP.

Lund JS, Henry GH, MacQueen CL, Harvey AR (1979) Anatomical organi- zation of the primary visual cortex (area 17) of the cat. A comparison with area 17 of the macaque monkey. J Comp Neurol 184:599-618.

Lund JS, Wu Q, Hadingham PT, Levitt JB (1995) Cells and circuits contributing to functional properties in area $\mathrm{V} 1$ of macaque monkey cerebral cortex: bases for neuroanatomically realistic models. J Anat 187:563-581.

Martin KAC (1988) From single cells to simple circuits in the cerebral cortex: the Wellcome prize lecture. Q J Exp Physiol 73:637-702.

Martin KAC, Whitteridge D (1984) Form, function and intracortical projections of neurones in the striate visual cortex of the cat. J Physiol (Lond) 353:463-504.

McGuire BA, Hornung JP, Gilbert C, Wiesel TN (1984) Patterns of synaptic input to layer 4 of cat striate cortex. J Neurosci 4:3021-3033.

Meskenaite V (1997) Calretinin-immunoreactive local circuit neurons in area 17 of the cynomolgus monkey, macaca fascicularis. J Comp Neurol 379:113-132.

O'Leary JL (1941) Structure of area striata of the cat. J Comp Neurol 75:131-164.

Peters A, Feldman ML (1976) The projection of the lateral geniculate nucleus to area 17 of the rat cerebral cortex. I. General description. J Neurocytol 5:63-84.

Peters A, Payne BR (1993) Numerical relationships between geniculocortical afferents and pyramidal cell modules in cat primary visual cortex. Cereb Cortex 3:69-78.

Peters A, Regidor J (1981) A reassessment of the forms of nonpyramidal neurons in area 17 of cat visual cortex. J Comp Neurol 203:685-716.

Peters A, Yilmaz E (1993) Neuronal organization in area 17 of cat visual cortex. Cereb Cortex 3:49-68.

Petersen CC, Sakmann B (2000) The excitatory neuronal network of rat layer 4 barrel cortex. J Neurosci 20:7579-7586.

Ramon y Cajal S (1988) Histology of the visual cortex of the cat. In: Cajal on the cerebral cortex (DeFelipe J, Jones E, eds), pp 495-523. Oxford: Oxford UP.

Somogyi P (1989) Synaptic organization of GABAergic neurons and GABAa receptors in the lateral geniculate nucleus and visual cortex. In: Neural mechanisms of visual perception (Lam DK, Gilbert CD, eds), pp 35-62. Houston: Portfolio Publishing.

Somogyi P, Cowey A (1981) Combined Golgi and electron microscopy study on the synapses formed by double bouquet cells in the visual cortex of the cat and monkey. J Comp Neurol 195:547-566.

Somogyi P, Freund TF, Cowey A (1982) The axo-axonic interneuron in the cerebral cortex of the rat, cat and monkey. Neuroscience 7:2577-2607.

Somogyi P, Kisvárday ZF, Martin KAC, Whitteridge D (1983) Synaptic connections of morphologically identified and physiologically characterized large basket cells in the striate cortex of the cat. Neuroscience 10:261-294.

Stratford KJ, Tarczy-Hornoch K, Martin KAC, Jack JJB (1996) Excitatory synaptic inputs to spiny stellate cells in the cat visual cortex. Nature 382:258-261

Szentagothai J (1973) Synaptology of the visual cortex. In: Handbook of sensory physiology: visual centers in the brain (Jung R, ed), Vol VII/3, pp 269-324. Berlin: Springer.

Szentagothai J (1978) The neuron network of the cerebral cortex: a functional interpretation. Proc R Soc Lond B Biol Sci 201:219-248.

Szentagothai J, Arbib MA (1974) Conceptual models of neural organization. Neurosci Res Prog Bull 12:313-510.

Tamás G, Buhl EH, Somogyi P (1997) Fast IPSPs elicited via multiple synaptic release sites by different types of GABAergic neurones in the cat visual cortex. J Physiol (Lond) 500:715-738.

Tamás G, Somogyi P, Buhl EH (1998) Differentially interconnected networks of GABAergic interneurons in the visual cortex of the cat. J Neurosci 18:4255-4270.

Thomas E, Patton P, Wyatt RE (1991) A computational model of the vertical anatomical organization of primary visual cortex. Biol Cybern 65:189-202.

Wahle P (1993) Differential regulation of substance P and somatostatin in Martinotti cells of the developing cat visual cortex. J Comp Neurol 329:519-538

Yabuta NH, Callaway EM (1998) Functional streams and local connections of layer $4 \mathrm{C}$ neurons in the primary visual cortex of the macaque monkey. J Neurosci 18:9489-9499.

Yabuta NH, Sawatari A, Callaway EM (2001) Two functional channels from primary visual cortex to dorsal visual cortical areas. Science 292:297-300. 